\title{
Wave atoms based compression method for fingerprint images
}

\author{
Zehira HADDAD ${ }^{1,2}$, Azeddine BEGHDADI ${ }^{1}$, Amina SERIR $^{2}$, Anissa MOKRAOUI $^{1}$ \\ ${ }^{1}$ L2TI, Institut Galilée, Université Paris 13, Sorbonne Paris Cité \\ 99 Avenue Jean-Baptiste Clément, 93430 Villetaneuse France \\ ${ }^{2}$ LTIR, Faculté d'électronique et d'informatique, USTHB, \\ BP 32 El Alia 16111 Bab Ezzouar Alger, Algérie
}

\begin{abstract}
This paper proposes a new fingerprint image compression approach where the quality of the decoded image is perceptually controlled using Wave atom transform. A comparative study of different transforms shows that Wave atom transform is the more appropriate than Wavelets for fingerprint image compression since it is able to better represent the geometrical structures of the fingerprint. A new image quality metric based on the same transform that has been used for compression is proposed to control the compression performance. Some properties of the human visual system are exploited and introduced in the developed metric. Simulations show that the proposed image quality metric correlates well with the subjective human judgment. According to these interesting results we developed a compression method specific to fingerprint images where the distortion is perceptually controlled. A recognition fingerprint system shows that the proposed strategy offers better results than traditional compression methods.
\end{abstract}

Keywords - Biometrics, Fingerprint compression, Image Quality Metric (IQM), Wavelets, Ridgelets, Curvelets, Wave atoms, Wavelet Scalar Quantization (WSQ), Human Visual System (HVS).

\section{Introduction}

During the last three decades, transform based image compression approaches have been extensively studied and some well-established standards for image and video coding appear since the 90's. Historically, many orthogonal transforms, such as the Discrete Fourier Transform (DFT), Haar Transform, Walsh Hadamard Transform, Slant Ttransform, the Discrete Cosine Transform (DCT) and some others interesting transforms have been used for lossy image compression [1]. The KarhunenLoeve Transform (KLT), also known as Hotelling Transform or Eigenvector Transform, is theoretically the best one, in the sense of energy compaction and decorrelation. However, it is data dependent and computationally more involved. For these main reasons KLT could not be used in practice [2].

A comparative study in [3] showed that one of the most suitable transform in terms of decorrelation and compactness is the DCT [4]. It offers the advantages of KLT without suffering from its drawbacks. Furthermore, unlike KLT, this transform uses a fixed basis, independent of the data. Some 
fast methods for calculating the DCT have been proposed [5]. For all these reasons, this transform have been adopted in JPEG and MPEG standards [2]. In JPEG compression scheme, the DCT is applied to blocks of $8 \times 8$ pixels. This results sometimes in a reconstructed image that has a blocky appearance when the compression ratio is high. This is one of the limitations that lead JPEG committee, as early as 1995, to develop a new Wavelet based compression standard for still images, namely JPEG 2000 [6], [7]. Indeed, Wavelets have received considerable attention in the last decades. This new standard offers better compression ratio while maintaining good image quality level without introducing annoying artefacts such as blocking effect in JPEG [2], [4], [7]. JPEG2000 has been also adopted as ISO and ANSI standard for biometric data exchange. This standard is dedicated to several types of images, but at low bit rate it may suffer from ringing and blurring effects. Some post processing solutions for reducing this artefact have been proposed in the literature [8], [9]. One possible solution is to introduce inside the coder artefact prediction and processing tool but at the expense of increased computational complexity. It would be then better to adopt the compression methods to the local structures of images. Many adaptive compression methods have been proposed in the literature [10]. Classical Wavelet transforms cannot effectively represent fine details in images for lacking of directionality. Some solutions have been then proposed to enhance the directional selectivity of Wavelets. The FBI fingerprint compression standard (Wavelet Scalar Quantization) WSQ is also based on Wavelet packet transform [7]. It has been reported that compression ratio attained by WSQ method ranges from 10:1 to $25: 1$ [29]. It is worth to notice that by taking into account some specific local and global structures of the fingerprint images the compression performance could be improved. Indeed, fingerprint images have specific geometric structures and fine details. Recently, many interesting multi-scale transforms have been developed. It has been shown that it is possible to define new multi-scale transforms more appropriate to the representation of geometric structures and abrupt transitions [10].

Fingerprint identification or authentication is often based on features extraction from local structures. An image quality is then of great importance for this purpose. Fingerprint image compression is prior to the recognition stage. Therefore, we need a quality measure that permits to adjust the compression independently to the extraction of local structures from fingerprints since this is done before and independently of the recognition stage. Figure 1 represents the proposed compression block diagram. Image quality has become a very active area of research during this last two decades. Indeed, the two image fidelity measures PSNR (Peak Signal to Noise Ratio) and MSE (Mean Square Error) are unfortunately the most used for the evaluation of image quality. These two metrics are simple, easy to compute and are mathematically convenient in the context of optimization on real time applications. However, it has been shown that they perform poorly in quantifying perceptual distortions. For example, small geometrical distortions may yield much higher MSE, without affecting the perceptual image quality. 
Several Image Quality Metric (IQM) somehow consistent with the human visual system (HVS) have been proposed [11]-[16], [17], [18]. Generally, image quality measures are listed in three main categories: Full Reference (FR), No Reference (NR) and Reduced Reference (RR).

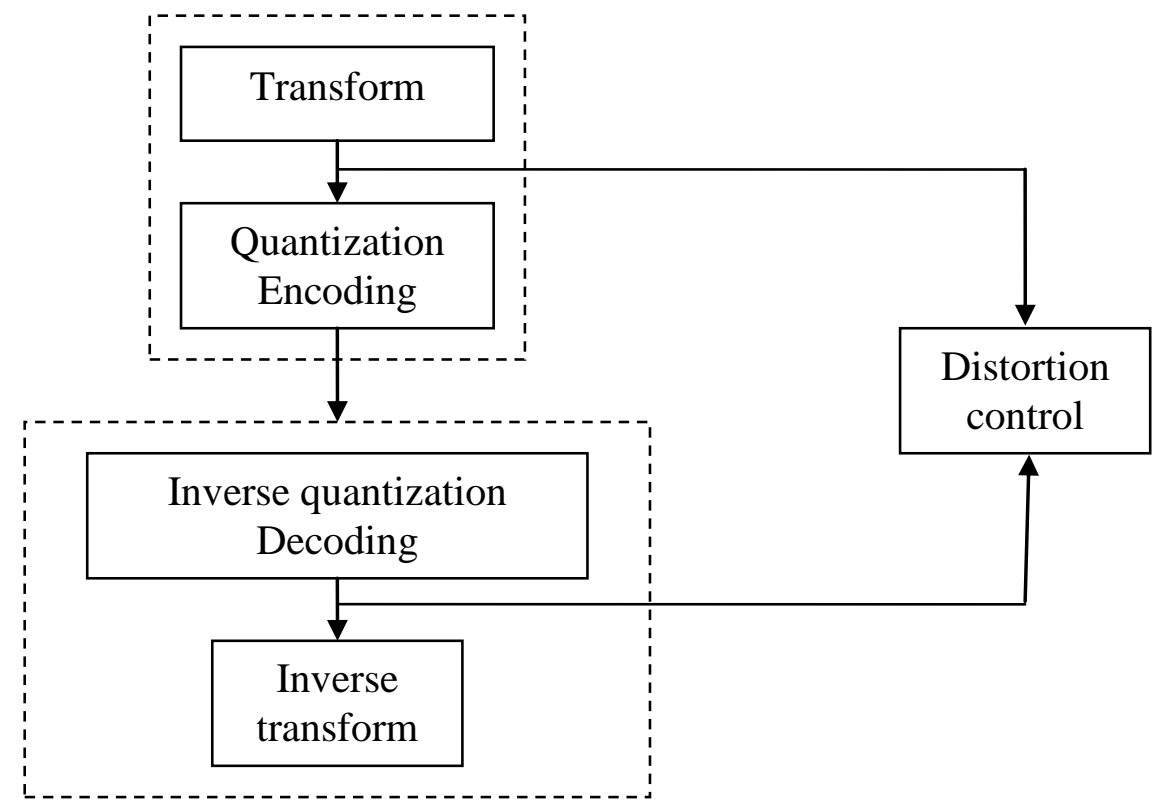

Figure1. Block diagram of the proposed compression system.

FR metrics use all the information of the reference image [19], [20], [21]. Many studies have focused on the use of the main properties of the HVS [11] in designing an image quality measure. The Visible Differences Predictor (VDP) proposed by Daly [19] is one of the approaches that are fully based on some perceptual characteristics of the HVS. But this kind of IQM is complex and time consuming. This limits its use in practice especially for real time applications. A new simple measure called MSSIM (Structural Similarity Index Method) using some implicit HVS properties has been proposed in [12]. It is based on the assumption that the HVS is sensitive to local structural information on the image. Its simplicity and efficiency in quantifying some degradation make it more attractive than PSNR or fully HVS based methods. The metrics which do not require any information about the original image belong to the no reference IQM category [13], [14], [15]. However, the main drawback of these metrics is that they are generally dedicated to specific degradation and cannot be generalized to evaluate any distortion.

As its name suggests, the last category of measures [16] RR IQM is a compromise between FR and NR IQM. These measures require some characteristics extracted from the original image.

Very recently, one of the geometric Wavelet transforms "Contourlet transform" has been used in the design of an image quality metric. The works in [17] and [18] offer two ways to use this transform for image quality assessment. The first is based on Contourlet transform and the second uses wavelet based Contourlet transform. By studying closely those new transforms, it appears that these 
geometrical transforms are very interesting and quite appropriate for the development of an image quality metric. These transforms offer a good representation of contours and fine details.

Other recent studies have focused on the introduction of image quality index to control the compression efficiency [23]. Among these studies, we quote JPEG2000 encoding with perceptual distortion control. This method is based on a model of vision which takes into account various masking effects of the human visual perception. The proposed metric incorporates spatial and spectral summation of individual quantization errors. The obtained results show that incorporating HVS model into JPEG2000 coder design significantly improves the JPEG2000 standard.

The aim of this work is now to show among the various new geometric Wavelets transforms, which is the most appropriate to fingerprint image compression. Then, we develop a control strategy of the compression using a new FR image quality metric which is based on the same transform. Finally, the performance of the proposed compression approach with distortion control is evaluated through a fingerprint recognition system.

This paper is organized as follows. Section 2 presents the background, including WSQ fingerprint compression standard and the different used geometric Wavelets transforms. Section 3 describes the proposed compression stage followed by the results of different compression experiments in order to choose the most adapted compression transform. The proposed image quality metric is introduced in section 4. Section 5 presents the strategy of the perceptual distortion control in the compression scheme and discusses the obtained results. Section 6 is devoted to the conclusion and perspectives.

\section{Background}

In the following we will emphasize the importance of considering the inherent features of fingerprint images when designing compression method. An efficient fingerprint compression method should be based on a good representation of the dominant fingerprint image structures. Indeed, the most important fingerprint image features are curves and oscillatory structures. In fact, the gray level along the curves of the fingerprint image reaches a minimum level corresponding to the contact with the sensor and then increases as one move away from contact with the sensor. Thus, the intensity profile representing the fingerprint epidermis relief can be considered as oscillatory patterns (Figure 2). The Wavelet transform is also considered in this study since the current image compression standard JPEG 2000 and the current fingerprint image compression standard WSQ are also based on this transform.

One way to better represent the fingerprint images is to optimize the Wavelet transform by considering the Wavelet packet decomposition and by maximizing the energy. This is the basic idea of WSQ standard. Thus, in this section, we review this compression standard dedicated to fingerprint images and we perform a prospective study of compression methods based on transforms that can better represent the important features of fingerprints. 


\section{IIIXI}

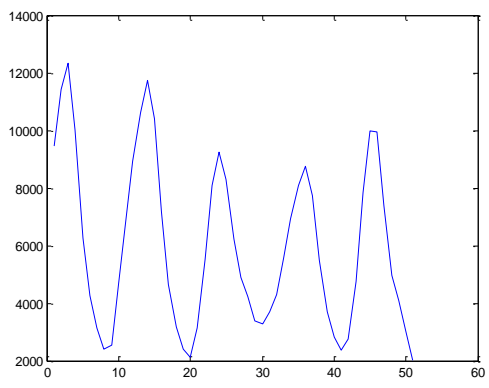

Figure 2. Local oscillatory curves structures of the fingerprint image.

\subsection{Wavelet Scalar Quantization standard}

The proliferation of biometric methods using fingerprints leads to an increase of the information to be stored in a dedicated database. For example the Federal Bureau of Investigations (FBI) fingerprint database contains many millions of fingerprint images. The use of lossy compression method is the only way to handle the huge size of this information. For this purpose, the FBI of USA has developed WSQ fingerprint image compression standard [7].

In Wavelet compression, the designer has the choice of the filter pair to use and which decomposition tree structure to follow. In WSQ scheme, several studies have been conducted in order to choose the most efficient Wavelet for fingerprint compression purpose [24], [25], [26], [27]. These studies have shown that the bi-orthogonal Wavelets are better than non bi-orthogonal ones for compression because the corresponding filters have the desirable property of being linear phase filters with minimum phase. In other terms, bi-orthogonal Wavelets do not exhibit frequency aliasing artifacts, while other Wavelets do. However, there are many bi-orthogonal Wavelets providing good compression results. An extensive study on Wavelet selection has been conducted on a very large set of wavelets [28]. This study revealed that out of the 4297 Wavelets tested, around 18 are efficient for compression [28]. Interestingly, the Wavelet specified in WSQ is among the 18 good wavelets. Furthermore, although the WSQ Wavelet was not the best, its filter lengths were the shortest and its performance was very close to the best Wavelet [28]. Generally, the structure of the decomposition tree can be determined dynamically during the compression stage but this is not the case in WSQ. The basic idea of a dynamic tree determination is to perform the tree decomposition according to the level of "energy" measured in each subband. If the energy level of a given subband is greater then a fixed threshold, this subband is transformed further, decomposed into smaller subbands. Then, the same energy test is 
applied on these elementary subbands. While this dynamic approach can yield higher compression ratio, its advantage is offset by the overhead of extra computational time to measure the energy level and the extra bits needed to represent the shape of the resulting decomposition tree. For this reason, in WSQ, another alternative was taken. This approach is to perform tests on several benchmark images in the intended application domain, such as fingerprints. From those tests, an appropriate optimal decomposition has been deduced. This has the advantage to avoid the overhead of dynamic decisions, while still producing nearly optimal results. The tests concluded that the best tree consists of 64 subbands [28]. In WSQ based compression method, each quantized subband is reshaped into one dimensional sequence, then Run Length Encoding (RLE) algorithm is applied to code runs of zeroes. Finally the run lengths and other remaining data are coded with Huffman algorithm.

\subsection{Geometric Wavelet transforms}

Wavelet transform has proven to be an efficient tool for image representation and analysis. The multiscale and localization properties of Wavelet make them a good candidate for image compression. Although Wavelets have been adopted in the current image compression standard JPEG2000, some limitations have been reported. Indeed, the extension of Wavelets in 2D domain is usually performed by a single separable tensor product, which generates multiple high-energy coefficients along the contours. In image processing, for reasons of simplicity and efficiency, Wavelets are often applied separately on the horizontal and vertical axis. This creates a partial decorrelation of the signal. Thus, it results in many highly energetic coefficients along the image contours. For example, orthogonal Wavelets have been proven more adapted for representing images containing regular areas, homogeneous textures and point singularities. But they can not exploit the regularity of geometric contours [30]. Although the effectiveness of Wavelets is well established for the representation of isotropic objects, their performance is rather limited in the case anisotropic objects such as lines and curves. To overcome this limitation, other transforms have been developed. In recent years, a new generation of multiscale and multidirectional transforms has been proposed to overcome these limitations [10].

For fingerprint compression, it would be efficient to search for new transforms requiring less information (fewer coefficients) to represent the geometrical structures. Geometric transforms seem to be good candidates for this purpose. There are two categories of geometric transforms, adaptive and nonadaptive transforms. Adaptive transforms use a geometric model of the image. Nonadaptive transforms or fixed transforms are based on directional filters banks, making them independent of the image content. Their advantage is that they do not require additional sequence during the synthesis stage in order to describe the analysis configuration. For all these reasons, we use nonadaptive transform approach in this paper. Among these transforms, Radon transform [31], Ridgelets [32], Curvelets [33], Contourlets [34], complex Wavelets [35], cortex transform [36] and steerable pyramid [37] are the most known. In the following, we recall some mathematical background to make the paper 
self sufficient.

\subsubsection{Radon, Ridgelet, Curvelet and Contourlet transforms}

Radon transform represents an image as a collection of projections along various directions [31], [38]. The directional properties of Radon transform make it the basis of many geometrical Wavelet transforms. The first geometrical transform based on Radon transform is the Ridgelet transform [32]. This last can be computed by performing a Wavelet analysis in the Radon domain, which leads to a multiscale and multidirectional analysis. However, Ridgelet transform has proven to be efficient for rectilinear discontinuities. Candes and Dohono [33] have developed a transform called Curvelet transform which is a simple generalization of Ridgelet transform for curvilinear contours. Indeed, the contours of an image are generally curvilinear. A curve can be represented by multiple linear segments. Based on this fact and that an image is supposed to contain locally linear contours, Ridgelet transform can be generalized to the curvilinear case. The basic idea of the first generation of Curvelet transform is then to represent an image as small parts of a certain size and a given orientation [33].

The first implementation of the Curvelet transform involves a multiscale image decomposition followed by a local Ridgelet transform [33]. However, this first generation of Curvelet transform presents some drawbacks. The spatial partitioning of this Curvelet generation uses overlapping windows to avoid blocking effects. Unfortunately, this leads to an increase of redundancy. To overcome this drawback a second generation Curvelet transform has been proposed [56].

A Curvelet of the second generation is defined as a function of $x$ at scale $2^{-j}$, orientation $\theta_{l}$ and position $x_{k}^{(j, l)}=R_{\theta, l}^{-1}\left(k_{1} 2^{-j}, k_{2} 2^{-j / 2}\right)$ by:

$\varphi_{j, l, k}(x)=\varphi_{j}\left(R_{\theta_{l}}\left(x-x_{k}^{(j, l)}\right)\right)$

Where $R_{\theta}$ is the rotation by $\theta$ radians. $\varphi_{j}$ is the waveform which represents a mother Curvelet such that all Curvelets at scale $2^{-j}$ are obtained by rotations and translations of $\varphi_{j}$.

The Curvelet coefficient is the inner product between an image $f$ and a Curvelet $\varphi_{j, l, k}$ defined by:

$$
c(j, l, k)=\left\langle f, \varphi_{i, l, k}\right\rangle=\int_{R^{2}} f(x) \varphi_{i, l, k}(x) d x
$$

The implementation of the second generation Curvelet transform consists of three main steps $(i)$ apply the 2D FFT to obtain the Fourier samples $\hat{f}\left[i_{1}, i_{2}\right]$;; (ii) for each scale $\mathrm{j}$ and angle $l$, compute the windowed frequency component and wrap it around the origin $\hat{f}\left[i_{1}, i_{2}\right] \hat{u}_{j, l}\left[i_{1}, i_{2}\right]$; (iii) compute the inverse 2D FFT to get the discrete Curvelet transform coefficients.

The windowing function $\hat{u}_{j, l}\left[i_{1}, i_{2}\right]$ gives rise to the frequency tiling shown in Figure 4-b [56].

Figure 3-a represents the spatial Cartesian grid associated with a given scale and orientation and figure 4-b represents the Curvelet tiling of frequency plane [56]. 


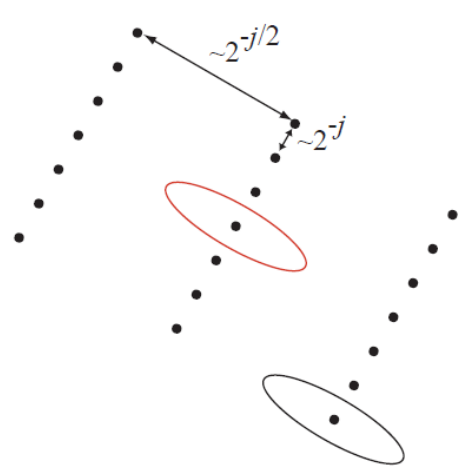

(a)

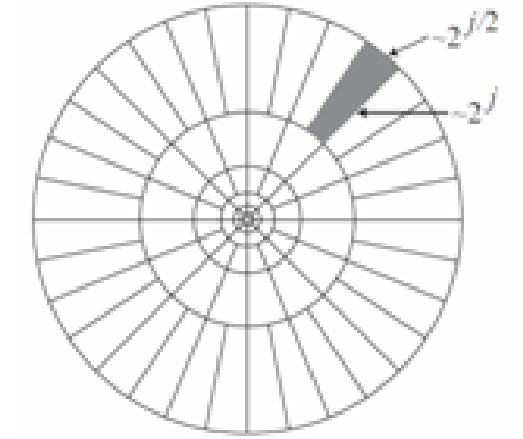

(b)

Figure 3. (a) Curvelet spatial Cartesian grid. (b) Curvelet tiling of frequency plane [56].

Contourlet transform is a transform which gives a comparable image analysis with Curvelet. But although Contourlet and Curvelet transforms seem to have the same properties, they differ in some aspects [34]. Contourlet transform unlike Curvelet transform uses the Laplacian pyramid for multiresolution aspect and directional filter banks for directional aspect.

All these properties made Curvelet and Contourlet transforms attractive for analyzing and representing fingerprint images. However, another important specificity for this type of image is the oscillatory aspect of the fingerprint patterns. Another geometric Wavelet transform, called Wave atom transform [39], seems to be very interesting for fingerprint image representation. In the following, we recall this transform.

\subsubsection{Wave atom transform}

Wave atom transform offers a better representation of images containing oscillatory patterns. This is the case of fingerprint images. This transform exhibits both the multiscale and the multidirectional properties.

To better understand and to situate Wave atoms transform among the other existing transforms, we follow the scheme analysis proposed in [39] where two indexes are introduced. The index $\alpha$ indicates whether the decomposition is multiscale $(\alpha=1)$ or not $(\alpha=0)$; and $\beta$ indicates whether basis elements are localized and poorly directional $(\beta=1)$ or, on the contrary, extended and fully directional $(\beta=0)$. Any adaptive transform consists to decompose an image in a database of functions defined in space by localization with width $2^{-\alpha j}$ and length $2^{-\beta j}$ and defined in frequency domain by a using a paving represented by the coordinates $2^{\alpha j}$ and $2^{\beta j}$ (Figure 4) [39]. 


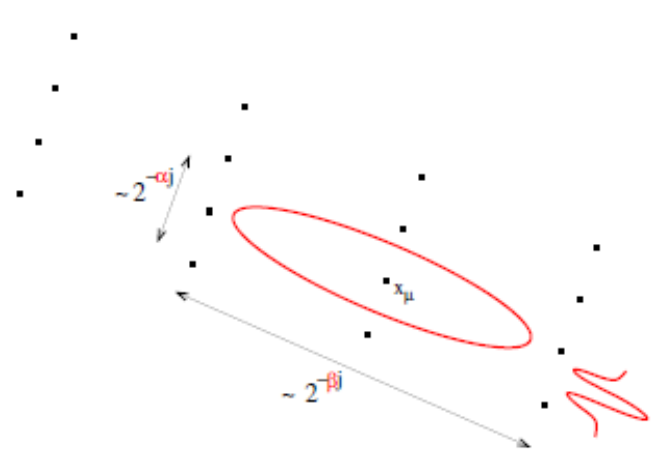

(a)

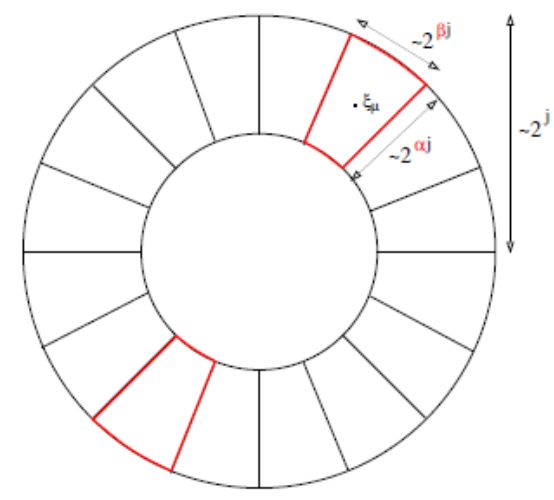

(b)

Figure 4. Adaptive transform tiling in (a) space and (b) frequency domains [39].

Following this classification: Wavelets (including multiresolution analysis [40], directional [41] and complex [42]) will correspond to $\alpha=\beta=1$, Ridgelet transform [32], to $\alpha=1, \beta=0$, Gabor transform to $\alpha=\beta=0$ and Curvelet transform [33] to $\alpha=1, \beta=\frac{1}{2}$. Wave atom transform is defined as a compromise between multiscale and multidirectional aspects with $\alpha=\beta=\frac{1}{2}$ (Figure 5).

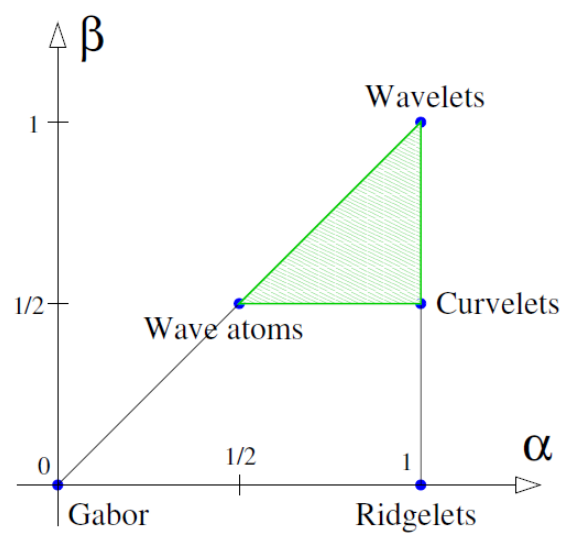

Figure 5. Classification of some modern harmonic analysis [39].

In the classical Wavelet transform, only the approximation is decomposed when passing from one subband to another. However, in the Wavelet packet, the decomposition can be pursued anywhere, in details and approximation bands, which is not optimal. Searching the optimality of the decomposition is like looking for the maximum energy of decomposition. This is the basis of wave forms.

Wave atom is implemented via the tensor products of adequately chosen 1D Wavelet packets [39]. Wave atom is noted $\phi_{\mu}(x)$, with subscript $\stackrel{\Delta}{\mu}(j, m, n) \stackrel{\Delta}{=}\left(j, m_{1}, m_{2}, n_{1}, n_{2}\right)$. The indexes $\left(j, m_{1}, m_{2}, n_{1}, n_{2}\right)$ are integer-valued associated to a point $\left(x_{\mu}, y_{\mu}\right)$ in the phase-space defined as follows: 
$x_{\mu}=2^{-j} n, \omega_{\mu}=\pi 2^{j} m, C_{1} 2^{j} \leq \max _{i=1,2}\left|m_{i}\right| \leq C_{2} 2^{j}$

Figure 6 represents the Wave atom tiling of the spatial frequency plane. When the scale $j$ increases by 1 , the square size doubles. At a given scale $j$, squares are indexed by $\left(m_{1}, m_{2}\right)$ starting from zero near the axis.

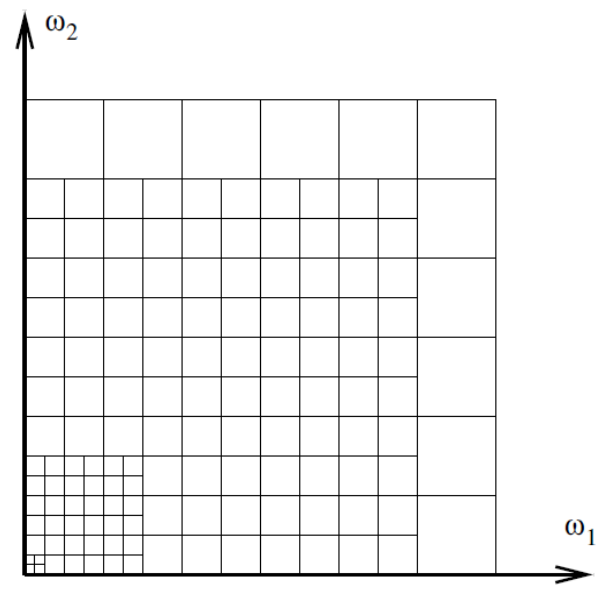

Figure 6. The Wave atoms tiling of the frequency plane [39].

\section{The proposed compression method}

It is worth noticing that the performance, in terms of energy compactness, sparsity, decorrelation and compression purpose, of any transform depends also on the image content. Thus, it is believed that the use of an appropriate transform for each type of image would help in providing effective tool to represent and analyze the main characteristics of the image. Here, we focus on the search of a transform which gives better compression results than classical Wavelet transforms. The aim of this work is not to compete with current compression standards dedicated to all types of images, but just to show that for certain type of image (such as fingerprint image), we can do better. It is well known that JPEG2000 compression standard performs quite well for a wide range of images. However, we demonstrate through this study that for a particular type of image, we could do better than JPEG2000. In the first part of this work, we perform a comparative study of the representation and compactness of different transforms. After this, we validate the compression results by applying the classic compression scheme using classical quantization and coding schemes [44].

\subsection{Representation and compactness: A comparative study}

For compression purpose, one of the objective criteria for selecting the most efficient transform is the quality reconstruction of the image from a set of limited transform coefficients. The best transform should be the one that gives the most faithful possible representation to the original using the smallest possible number of coefficients. Then, we search for the most parsimonious and compact 
representation of the image.

For this purpose, we use a large set of images and different transforms. The fingerprint image contains many curves and contours. From the previous study on the state-of-the-art, it appears that some transforms are good candidates for fingerprint compression: Wave atoms, Curvelet, Contourlet and the classical Wavelet transform. The interesting properties of Curvelet and Contourlet transformations and especially their ability in capturing and representing the geometric structures in the image [33] make it good candidates for this preliminary study. We do not consider Ridgelet transform since Curvelet transform is a generalization of Ridgelet and it has been proven more efficient for capturing the geometric structures. We have also chosen the Wave atom transform for its interesting properties such as the type of decomposition "half multi-scale and half multi-directional" and the good representation of images containing oscillatory patterns such as fingerprints [39].

We use different types of images: medical images (x ray lung and skull MRI), satellite images (bay view and city built view), texture images (grass and pattern texture), fingerprint images (arc and loop) and other classical test images like Lena, Barbara, peppers, house, Flintstones and boat. Figure 7 represents the image database which has been used.

In order to evaluate the image quality, we calculate the PSNR. Table 1 presents for these different types of images, the mean values of PSNR as a function of the percentage of selected input coefficients. This percentage refers to the input image size. Note that we use here the orthogonal version of Wave atom transform which is non-redundant. For all the used transforms, we decompose into five frequency subbands.

The procedure consists of four steps:

1) Decompose the original image by using different transforms.

2) Store the coefficients for each transform, from highest to lowest.

3) Apply different thresholds on the number of coefficients in order to keep only the coefficients that best represent the image (the coefficients of the highest absolute value).

4) Reconstruct the image with the selected number of coefficients and measure the image quality compared to the original.

We used the free toolboxes Curvlab 2.0.2, Contourlet Toolbox 2.0 and WaveAtom1.1 available at the websites: curvelet.org, http://www.ifp.uiuc.edu/ minhdo/software and waveatom.org, respectively.

The obtained results demonstrate that generally for the same number of coefficients, the Wavelet transform gives the best PSNR except for fingerprint images where Wave atoms transform is better. Through these results, it is demonstrated that Wave atom transform is the most suitable transform for fingerprint images representation and compression.

The results of Table 1 were confirmed on the fingerprint images database FVC 2004 [45]. This database contains 80 fingerprint images. Figure 8 represents the mean values of the PSNR obtained for images of the database as a function of the percentage of selected coefficients for the four considered transforms: Wave atom, Wavelet, Curvelet and Contourlet. 
Simulations have been performed with Matlab on Toshiba personal computer Intel Core 2Duo CPU T8100@2.10 GHz and 3 Go RAM. The decomposition computational time measured for each transform is as follows. When the elapsed time for Wavelet is $0.20 \mathrm{~s}$, the elapsed times are $0.60 \mathrm{~s}, 2.45 \mathrm{~s}$ and $2.45 \mathrm{~s}$ for Wave atom, Curvelet and Contourlet, respectively.
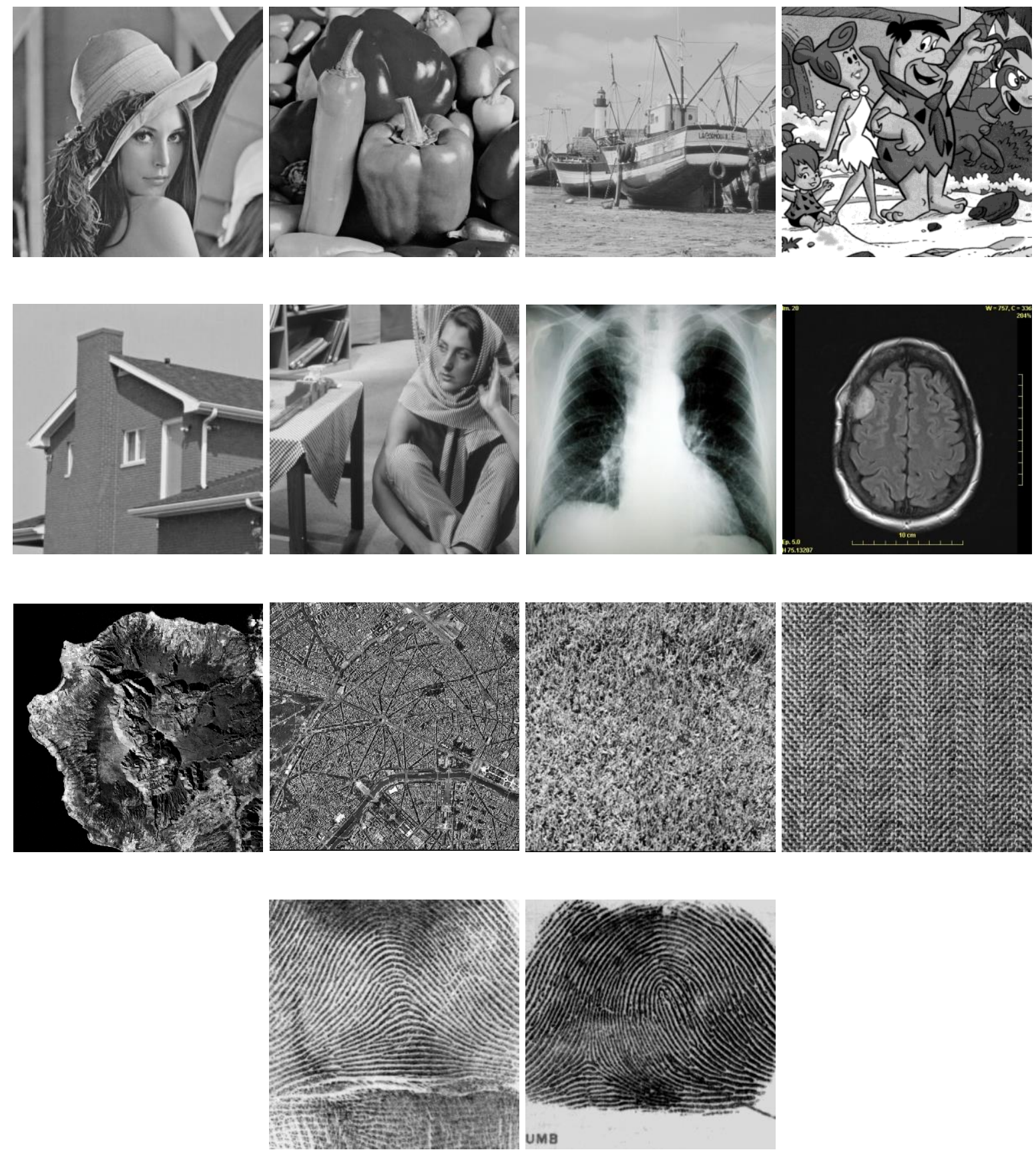

Figure 7. Image database. 


\begin{tabular}{|c|c|c|c|c|c|c|}
\hline & \multirow{2}{*}{ Transform } & \multicolumn{5}{|c|}{ Percentage of selected coefficients } \\
\hline & & $10 \%$ & $20 \%$ & $30 \%$ & $40 \%$ & $50 \%$ \\
\hline \multirow{4}{*}{ 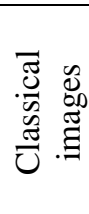 } & Wave atom & 27.07 & 29.74 & 31.97 & 34.12 & 35.62 \\
\hline & Wavelet & 29.65 & 34.78 & 38.53 & 41.78 & 44.93 \\
\hline & Curvelet & 26.53 & 31.25 & 34.85 & 38.21 & 41.65 \\
\hline & Contourlet & 25.72 & 28.68 & 31.49 & 33.15 & 35.54 \\
\hline \multirow{4}{*}{ 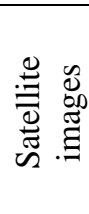 } & Wave atom & 18.72 & 20.04 & 20.88 & 22.49 & 23.09 \\
\hline & Wavelet & 17.84 & 21.51 & 23.78 & 26.51 & 29.62 \\
\hline & Curvelet & 18.48 & 19.64 & 20.89 & 23.54 & 26.20 \\
\hline & Contourlet & 18.38 & 21.01 & 22.46 & 25.36 & 26.74 \\
\hline \multirow{4}{*}{ 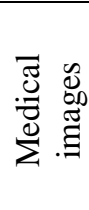 } & Wave atom & 32.57 & 34.33 & 35.41 & 36.91 & 39.42 \\
\hline & Wavelet & 38.95 & 42.14 & 46.84 & 48.84 & 52.83 \\
\hline & Curvelet & 30.42 & 37.43 & 39.87 & 43.27 & 46.02 \\
\hline & Contourlet & 24.18 & 28.61 & 32.40 & 36.39 & 39.17 \\
\hline \multirow{4}{*}{ 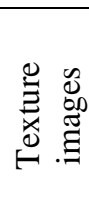 } & Wave atom & 21.25 & 22.87 & 24.12 & 26.41 & 27.58 \\
\hline & Wavelet & 21.02 & 24.17 & 26.83 & 28.98 & 31.67 \\
\hline & Curvelet & 19.54 & 21.56 & 24.47 & 26.52 & 29.14 \\
\hline & Contourlet & 22.25 & 24.65 & 26.84 & 28.00 & 31.23 \\
\hline \multirow{4}{*}{ 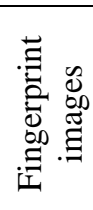 } & Wave atom & 25.61 & 27.72 & 30.64 & 33.64 & 35.35 \\
\hline & Wavelet & 22.65 & 26.33 & 28.71 & 30.47 & 34.60 \\
\hline & Curvelet & 22.64 & 25.53 & 28.60 & 30.27 & 33.48 \\
\hline & Contourlet & 21.40 & 24.55 & 27.74 & 29.90 & 31.96 \\
\hline
\end{tabular}

Table 1. Mean Values of PSNR vs the percentage of selected coefficients for natural images.

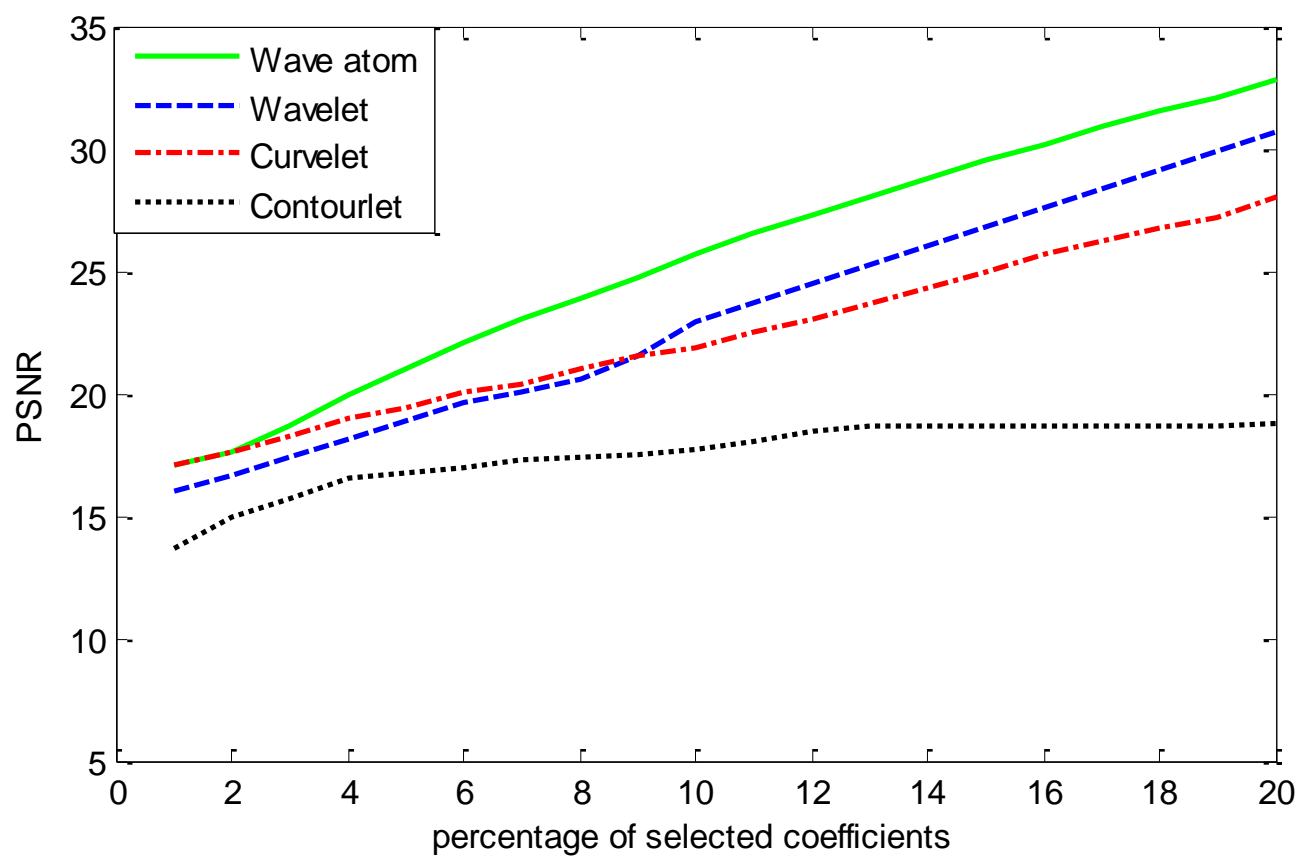

Figure 8. Mean values of PSNR vs the percentage of selected coefficients for fingerprint images database. 


\subsection{Compression performances evaluation}

To evaluate the efficiency of Wave atom for fingerprint image compression, we integrate it in the whole compression chain. The main three steps of any transform based compression method, namely, transform, quantization and coding, are used.

We use a uniform scalar quantization with dead zone instead of vector quantization. Indeed, previous works [7] demonstrated that in practice, the decomposition tree is so profound that the size of the low frequency subband is so small that no significant advantage could be gained from the vector quantization. Furthermore, for the other subbands (high frequency), when the decomposition is correctly chosen, the coefficients of all subbands are highly uncorrelated, and thus the use of vector quantization yields an important overhead/

Since many nulls coefficients are engendered by the Wave atom transform and quantization process. Here, each quantized subband is first converted into a one-dimensional sequence, and then RLE is used to encode the sequence of zeros. The sequence length and the remaining data are then encoded with the Huffman algorithm. This strategy has been also used in WSQ fingerprint image compression standard [7].

Figure 9 presents the Rate-Distortion curves concerning fingerprint image compression using the three transforms: Wave atoms, Curvelets and Wavelets. In order to perform an objective comparison between the different transforms, we apply the same quantization and coding strategy.

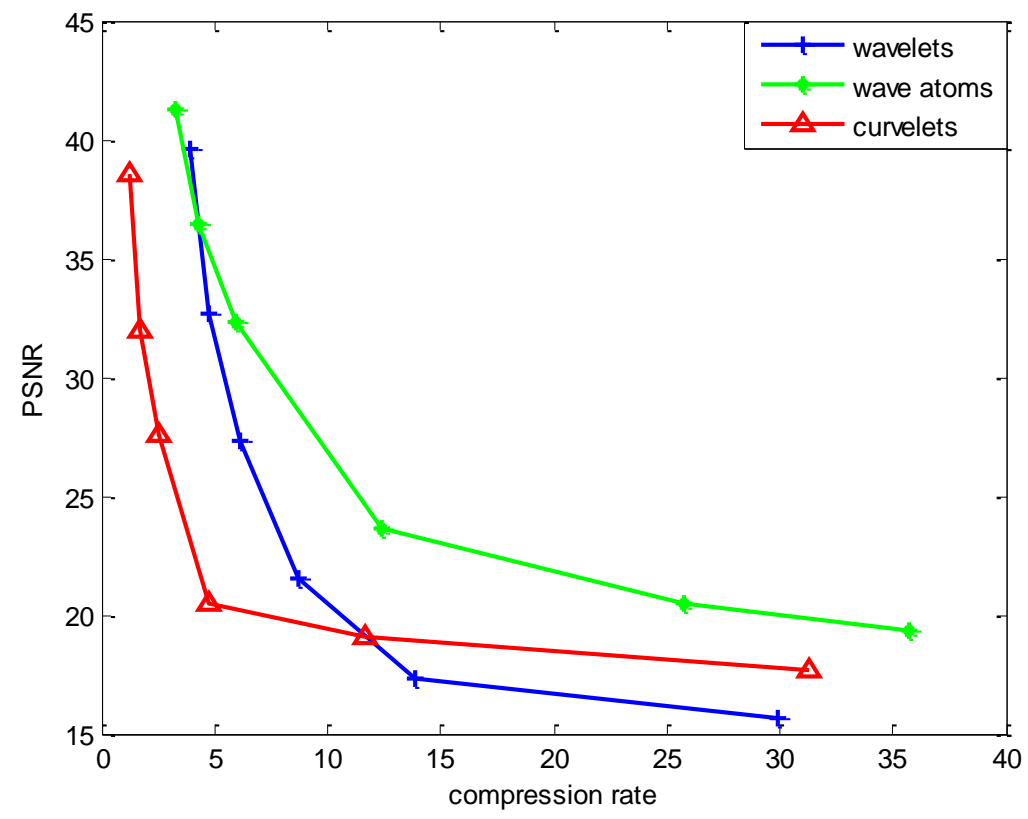

Figure 9. Rate-distortion curves for fingerprint image using Wave atoms, Curvelets and Wavelets. 
For the same compression ratio 15:1, the original image (a) and the different images resulting from the three compression methods using Wave atoms (b), Wavelets (c) and Curvelets (d) are shown in Figure10. The decoded image based on Wave atom transform does not introduce any visible degradation unlike the Wavelet transform where a visible blocking effect appears. For the Curvelet transform, we notice a smoothing effect on many regions of the image. These results show that Wave atom transform outperforms the other tested compression techniques.

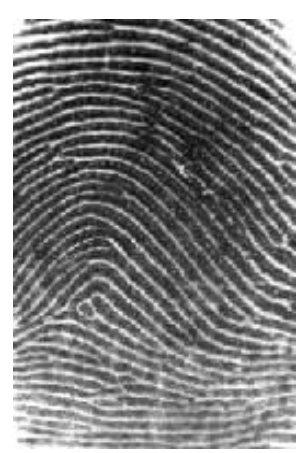

(a)

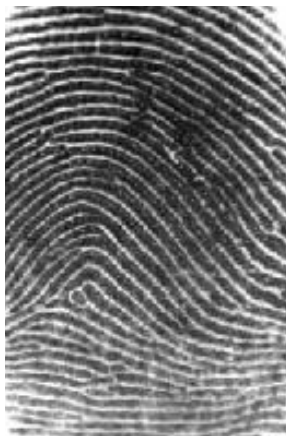

(b)

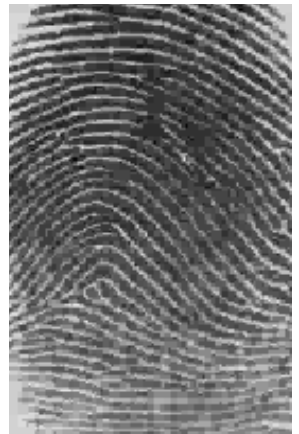

(c)

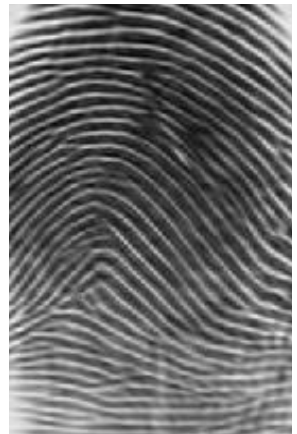

(d)

Figure 10. (a) Original image, decoded image using (b) Wave atoms, (c) Wavelets and (d) Curvelets.

\section{The proposed image quality metric}

The estimation of the distortion level resulting from the compression of a fingerprint image is a useful step for controlling the compression process. We propose then, a new FR image quality metric based on Wave atom transform that exploits some properties of the human visual system, in order to make it consistent with subjective evaluation. It is based on more advanced analysis and representation tools to extract more relevant features that could be incorporated in the design of this metric. It is well known that the main features of fingerprint images are minutiae. However, these minutiae are extracted after the compression process. The compression is performed independently to the using recognition algorithm and should not affect its efficiency. For this reason, we do not use minutiae to control the compression ratio and we develop a new image quality metric, which reflects the quality perception of fingerprint images. This IQM must be in agreement with the recognition process. A good image quality metric should be able to capture the key structural features and the artifacts or distortions that may result from the compression or transmission. In some interesting works, authors attempt to define the quality of biometric data and especially fingerprint images by exploiting the local structure of minutia. But all the proposed metrics are based only on the local structures of the minutia and do not consider some artifact that may result from the compression process [60], [61]. For this purpose, Wave atom appears as a good candidate for evaluating the compression effect on fingerprint images. The use of a perceptual image quality metric based on wave-atom in order to control the compression process is dictated by three objectives. First, we want a fully wave-atom based compression system with image 
quality control. Second, we want to put more strength on the image quality to ensure the highest level of quality control and recognition rate. Third, the perceptual image quality of any biometric data may influence in one way or another recognition system.

\subsection{Description of the proposed image quality metric}

The developed image quality metric consists of three important steps namely, extraction of the representative Wave atom coefficients; application of the HVS based masking effect and finally the computation of a single value representing the image quality level.

First, we extract from the original image $I_{R}$ and the distorted image $I_{D}$ the respective Wave atom coefficients $c_{l, o}^{R}(x, y)$ and $c_{l, o}^{D}(x, y)$ corresponding to the point $(x, y)$ in the subband $(l, o), l$ representing the scale and $o$ the orientation. Then, we apply a perceptual masking model in order to take into account the fact that the visibility of one image component (the target) is reduced by the presence of another (the background). Watson et al [46] showed that image quality models should incorporate entropy masking, as well as contrast masking. Contrast masking permits to take into account the modification of the visibility threshold due to the contrast value. Entropy masking allows to consider the visibility threshold modification due to the neighborhood characteristics.

Here, we use a semi-local masking model inspired by Daly's model [47]. For each site $(x, y)$ at the scale $l$ and the orientation $o$, we calculate the visibility threshold elevation $T_{l, o}(x, y)$ following [18]:

$T_{l, o}(x, y)=\left(1+\left(k_{1} \cdot\left(k_{2} \cdot\left|c_{l, o}(x, y)\right|\right)^{S+\Delta s(x, y)}\right)^{b}\right)^{1 / b}$

$k_{1}, k_{2}$ correspond to the pivot point of the contrast curve.

$b$ determines how closely the curve follow the asymptote in the transition region.

$s(x, y)=S+\Delta s(x, y)$ represents the slope of the high masking contrast asymptote, depending on the neighborhood.

$\Delta s(x, y)$ is calculated from the entropy on a neighborhood of the tested image as follows:

$\Delta s(x, y)=\frac{b_{1}}{1+e^{-b_{2}\left(E(x, y)-b_{3}\right)}}$

$E(x, y)=-\sum p(x, y) \log (p(x, y))$

$E(x, y)$ is the resulting entropy map associated with the probability $p(x, y)$ derived from the luminance distribution of the neighborhood around site $(x, y)$.

$b_{1}, b_{2}, b_{3}$ are adjustable parameters.

Here, as in [18], we use the following values:

$b_{1}=0.3 ; b_{2}=2 ; b_{3}=1 ; k_{1}=k_{2}=1 ; b=2 ; S=0.65$

After this step, we normalize the error between the Wave atom coefficients of the original image and 
the distorted image using the following equation:

$$
N E_{l, o}(x, y)=\frac{\left|c_{l, o}^{R}-c_{l, o}^{D}\right|}{\max \left(T_{l, o}^{R}(x, y), T_{l, o}^{D}(x, y)\right)}
$$

In order to obtain a single value representing the image quality, we combine the different information from each location, scale and orientation subbands as follows:

$$
\begin{aligned}
& N E_{l, o}=\left(\frac{1}{X_{l, o} \cdot Y_{l, o}} \sum_{x=1}^{X_{l, o}} \sum_{y=1}^{Y_{l, o}}\left(N E_{l, o}(x, y)\right)^{2}\right)^{1 / 2} \\
& N E=\frac{1}{L} \sum_{l=1}^{L}\left(\frac{1}{O_{l}} \sum_{o=1}^{o_{l}} N E_{l, o}\right)
\end{aligned}
$$

Finally, the proposed Wave Atom based image quality Metric (WAM) is given by:

$$
W A M=\log _{10}(N E+1)
$$

\subsection{Evaluation of the proposed image quality metric}

We recall that the goal of any IQM is to make the quality measure consistent with subjective evaluation of human observers. We use for our experiments two IQM databases, the LIVE database [48] and the IVC database [57]. These databases contain a large data set of images with various degradations and associated MOS (Mean Opinion Score).

LIVE database contains five degradations due to JPEG2000 compression, JPEG compression, White Noise (WN), Gaussian Blur (GB), and bit errors in JPEG2000 bitstream when transmitted over a simulated Fast-Fading Rayleigh channel (FF). For evaluation criteria, we use: Pearson linear Correlation Coefficient (PCC) and Spearman Rank-Order Correlation Coefficient (ROCC).

A logistic function is used to adjust the objective image quality metric outputs and model it by a curve using a non-linear regression method [18]. This regression is done by minimizing the mean square error between the proposed image quality metric measures and subjective measures. Figure 11 illustrates these results. We note that the data are not very scattered, which proves that the image quality metric is consistent with the mean opinion scores.

Table 2 summarizes the obtained results with WSSIM [49], WBCT [50], MSDD [18] and the proposed metric WAM. Note that the proposed metric gives the highest correlation coefficient for all considered distortions. These results prove the efficiency of the WAM in predicting the distortion level. This performance is mainly due to the use of Wave atom transform, where the loss of fidelity are analyzed using an appropriate multiscale and multidirectional approach combined with some HVS characteristics such as masking effect. 
(a)JPEG2K

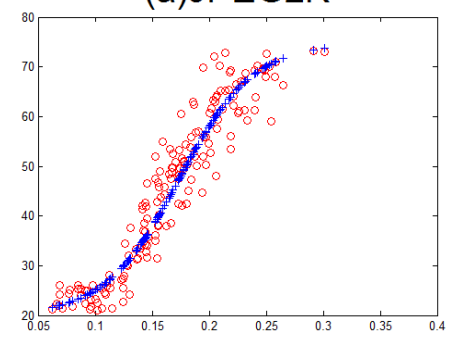

(b)JPEG

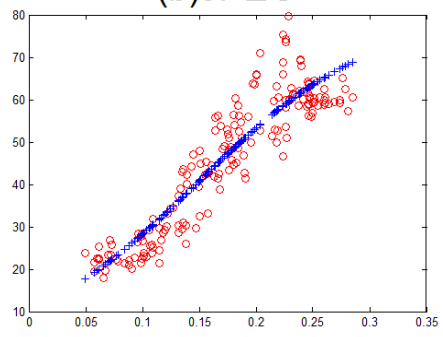

(c)WN

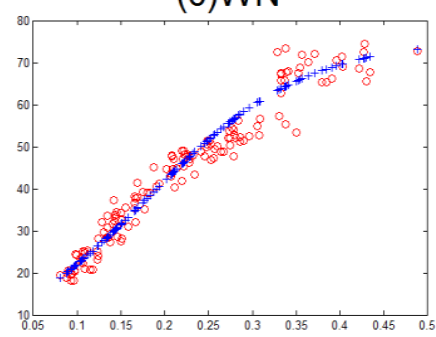

(e)FF

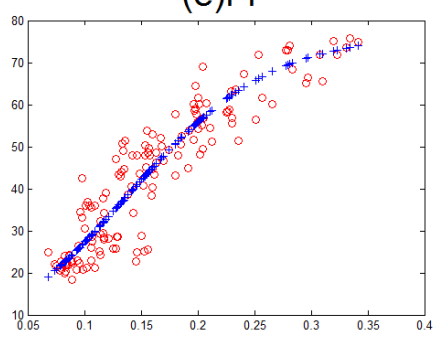

Figure 11. Subjective ratings of the perceived distortion for different classes of the LIVE database as function of the predicted values by the proposed IQM. In all graphs, the vertical axis denotes DMOS

(Difference Mean Opinion Score). The horizontal axis corresponds to the proposed metric.

\begin{tabular}{|c|c|c|c|c|c|c|}
\hline \multicolumn{7}{|c|}{ Pearson Correlation Coefficient : PCC } \\
\hline & JPEG2k & JPEG & WN & GB & FF & All \\
\hline WAM & 0.980 & 0.971 & 0.980 & 0.971 & 0.956 & 0.933 \\
\hline WSSIM & 0.940 & 0.935 & 0.962 & 0.952 & 0.953 & 0.884 \\
\hline WBCT & 0.914 & 0.880 & 0.970 & 0.377 & 0.812 & 0.674 \\
\hline MSDD & 0.942 & 0.940 & 0.984 & 0.959 & 0.919 & 0.890 \\
\hline \multicolumn{7}{|c|}{ Spearman Rank Order Correlation Coefficient: ROCC } \\
\hline WAM & 0.979 & 0.957 & 0.988 & 0.973 & 0.956 & 0.949 \\
\hline WSSIM & 0.931 & 0.899 & 0.957 & 0.960 & 0.962 & 0.879 \\
\hline WBCT & 0.919 & 0.825 & 0.979 & 0.312 & 0.782 & 0.624 \\
\hline MSDD & 0.936 & 0.904 & 0.978 & 0.958 & 0.916 & 0.904 \\
\hline
\end{tabular}

Table 2. Correlation results with LIVE database.

IVC database [57] uses 10 original images and 235 degraded images. It includes different types of degradation such as JPEG and JPEG 2000, "LAR coding" and blurring. The evolution of the IVC database subjective ratings as function of the proposed image quality metric is shown in Figure 12. The shape of the scatter plot is not very dispersed. It shows that the proposed metric is globally in agreement with the MOS. 
(a) JPEG2000

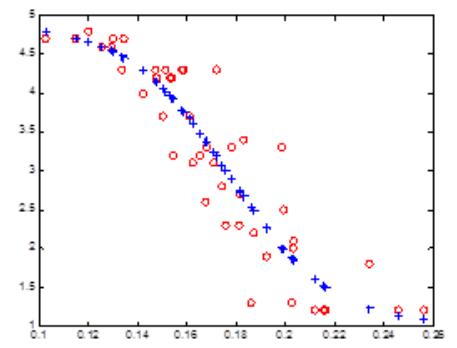

(b)JPEG

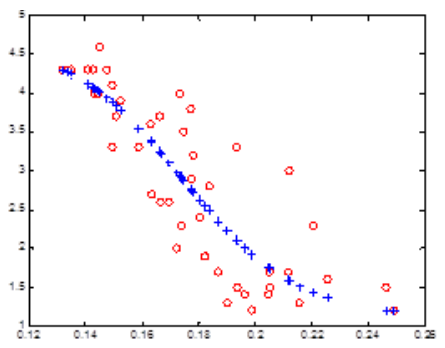

(c)LAR

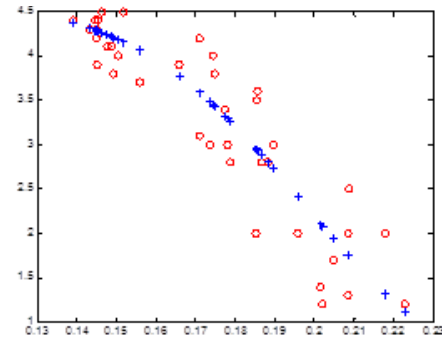

(d)JPEG_lumi chr

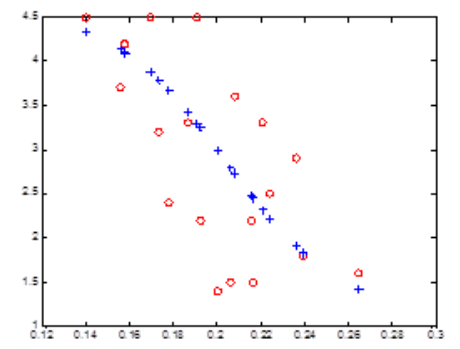

(e)BLUR

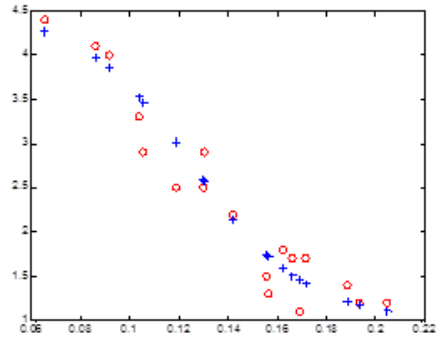

Figure 12. Subjective ratings of the perceived distortion for different classes of the IVC database as function of the predicted values by the proposed IQM. In all graphs, the vertical axis denotes MOS

(Mean Opinion Score). The horizontal axis corresponds to the proposed metric.

Table 3 presents the obtained results for this database compared with SSIM [12] and VSNR [58]. The results confirm the previous results. The proposed IQM is highly correlated with subjective appreciations.

\begin{tabular}{|c|c|c|c|c|c|}
\hline \multicolumn{7}{|c|}{ Pearson Correlation Coefficient PCC } \\
\hline & JPEG2k & JPEG & LAR & JPEG_lumichr & BLUR \\
\hline WAM & $\mathbf{0 . 9 1 8 0}$ & $\mathbf{0 . 8 5 7 9}$ & $\mathbf{0 . 9 0 4 1}$ & $\mathbf{0 . 6 7 1 7}$ & $\mathbf{0 . 9 6 5 7}$ \\
\hline SSIM & 0.8050 & 0.7419 & 0.6354 & 0.6290 & 0.8681 \\
\hline VSNR & 0.8352 & 0.7929 & 0.7129 & 0.6283 & 0.9641 \\
\hline \multicolumn{7}{|c|}{ Spearman Rank Order Correlation Coefficient: ROCC } \\
\hline \multicolumn{7}{|c|}{ JPEG2k } & JPEG & LAR & JPEG_lumichr & BLUR \\
\hline WAM & $\mathbf{0 . 9 1 8 7}$ & $\mathbf{0 . 8 6 4 4}$ & $\mathbf{0 . 8 9 3 9}$ & 0.6456 & 0.9224 \\
\hline SSIM & 0.8315 & 0.7898 & 0.6603 & $\mathbf{0 . 6 7 4 2}$ & 0.8407 \\
\hline VSNR & 0.8381 & 0.7843 & 0.7002 & 0.6218 & $\mathbf{0 . 9 5 6 6}$ \\
\hline
\end{tabular}

Table 3. Correlation results with IVC database.

\section{The proposed image compression with distortion level control}

Given the diversity of fingerprint images for a given compression level, different quality levels could be obtained for each image. In other words, for a given quality level, we can compress more one fingerprint image than another one. The goal of this work is to compress different fingerprint images at different compression ratios while maintaining a fixed image quality level. 
The design of an efficient lossy compression system dedicated to fingerprint should be able to preserve the image quality to avoid any negative impact on the recognition system. The idea is to introduce in the system a quality control mechanism which allows to adjust the compression ratio according to the desired image quality level. We recall that minutiae detection could not be used as a criterion for controlling the compression ratio, since it depends on the used recognition approach. Recent decades have seen the emergence of different methods that provide good recognition results without using any minutiae matching process [51], [52], [53]. Moreover, the security systems like FBI, store fingerprint images as they are, just after the acquisition stage.

The principal interest of this approach is that it is based on a transform suited to fingerprint images. The two modules of compression and image quality control are both based on this transform, which presents a significant advantage in terms of flexibility and homogeneity.

\subsection{Compression performance evaluation}

In our experiments, we compress fingerprint images at different compression ratios ranging from 25:1 to $182: 1$ with the proposed Wave atom compression method using an adaptive uniform scalar quantization with dead zone and RLE combined with Huffman coding. Quantizer is adaptive within the subbands in the sense that the length of the quantization steps is inversely proportional to the logarithm of the variance in the subband.

To compare the proposed compression method to the most successful ones, we also present the same fingerprint images compressed at the same compression ratios with JPEG2000 image compression standard, WSQ FBI standard and SPIHT. The comparison with the basic mode of JPEG2000 corresponding to Part I is available at [54]. This part uses a scalar quantization with dead zone and arithmetic coding. The comparison of the different compression methods is performed using PSNR, and the proposed image quality metric WAM. The IQM value is low when the distortion rate is low and this corresponds to better image quality. This in contrast with PSNR, higher is the PSNR, better is the image quality.

Figure 11 represents the rate distortion curves corresponding to these images and the considered compression methods. This figure presents the PSNR as function of the compression ratio. Figure 12 shows the plot of the proposed image quality measure WAM as function of the compression ratios. Figure 13 illustrates that the highest PSNR corresponds to Wave Atom Compression (WAC) scheme. Similarly, Figure 14 shows that the lowest distortions correspond to the proposed WAC method.

To evaluate the performance of the proposed approach, we conducted a set of experiments on several fingerprint images of different kinds. Generally, the fingerprint images are classified into pre-specified classes according to their content. Figure15 illustrates the obtained results for each fingerprint class (one fingerprint image compressed at different compression ratios per class). 


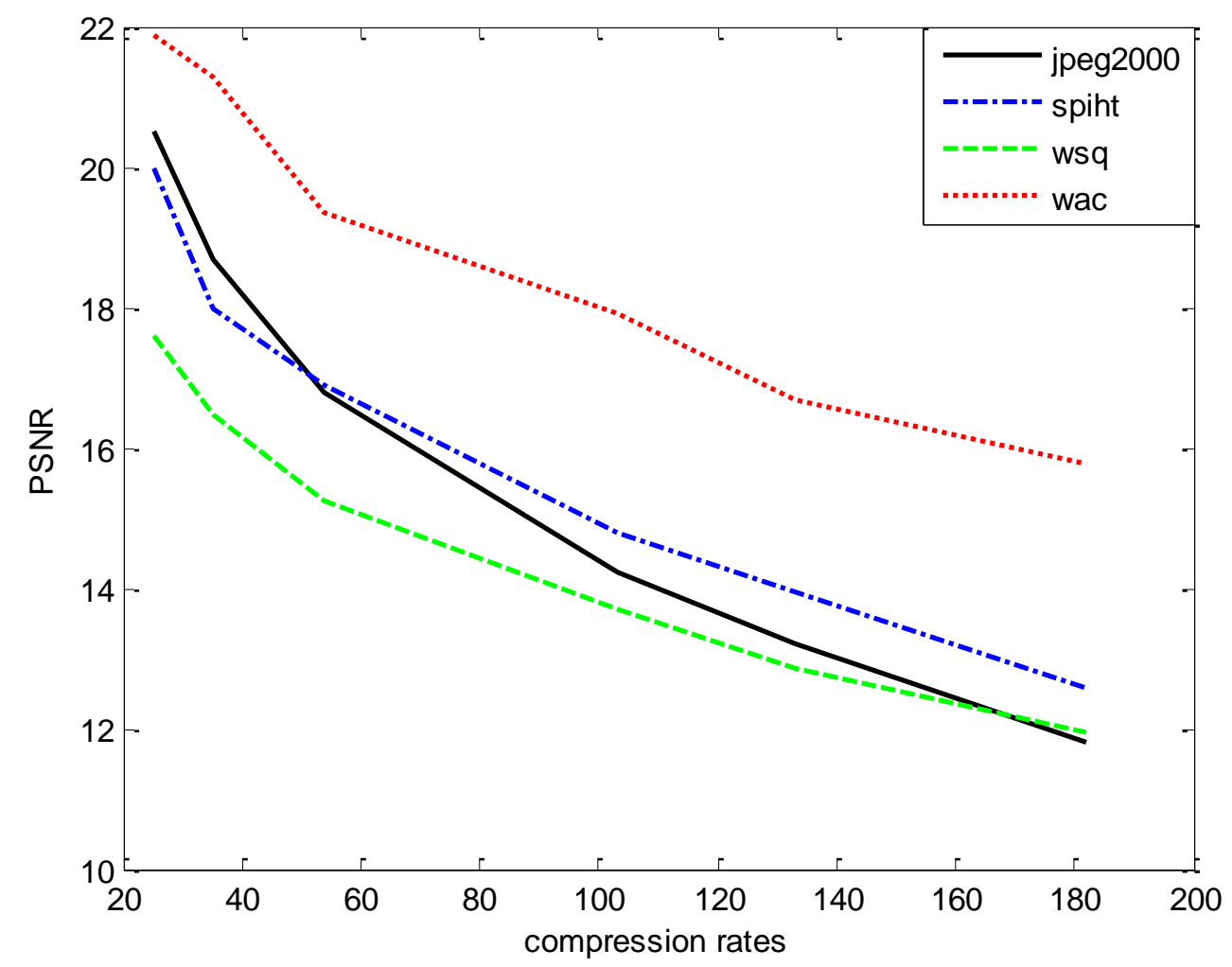

Figure 13. Rate-distortion curves: PSNR as function of compression rates for fingerprint image using JPEG2000, WSQ, SPIHT and Wave Atom based compressions.

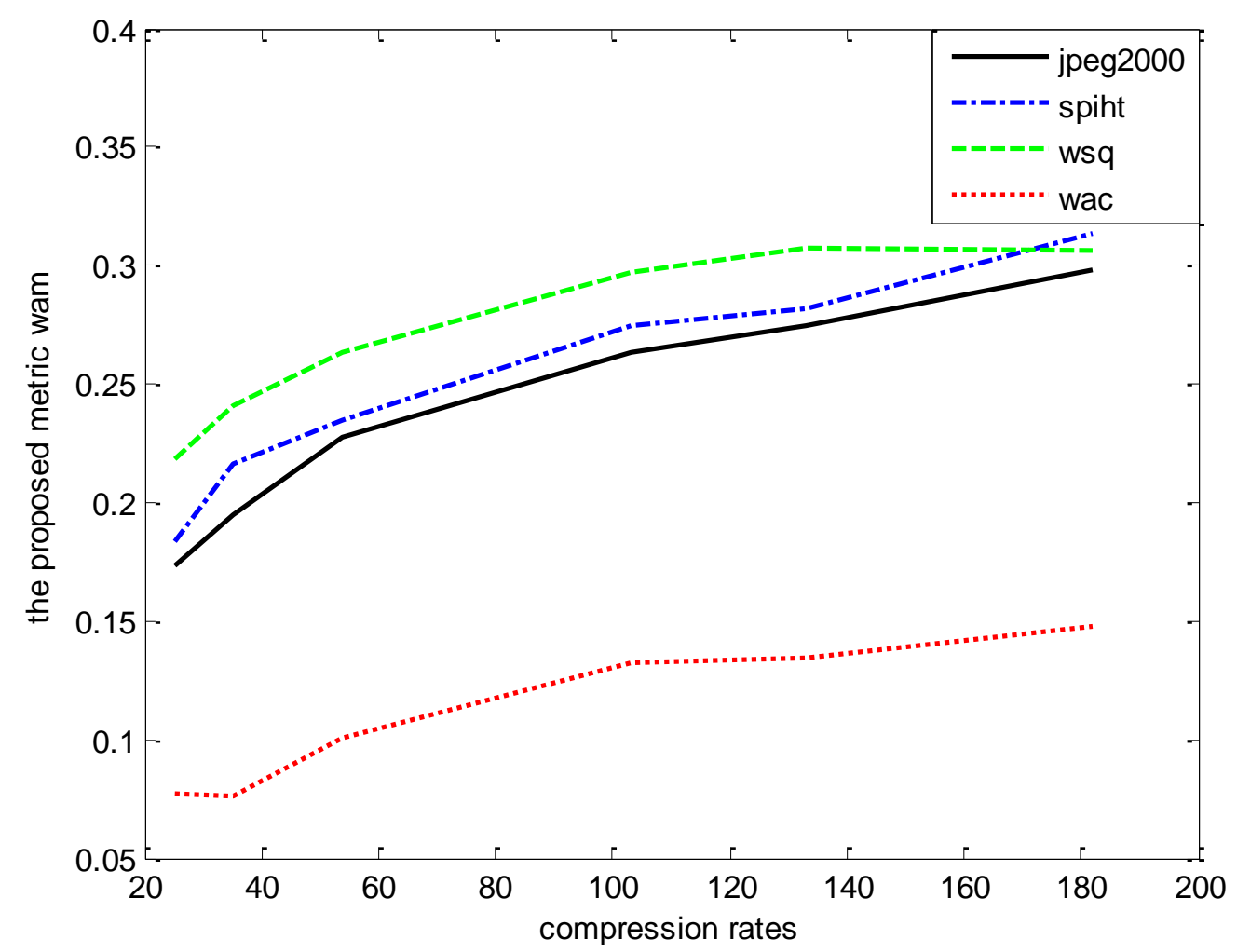

Figure 14. Rate-Distortion curves: WAM (measure of degradation) as a function of compression ratio for fingerprint image using JPEG2000, WSQ, SPIHT and WAC. 


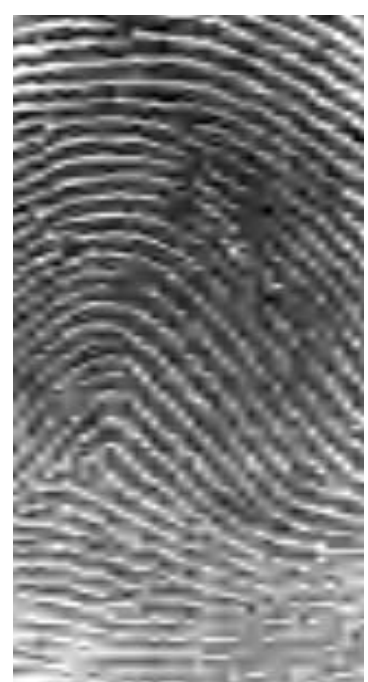

(a1)

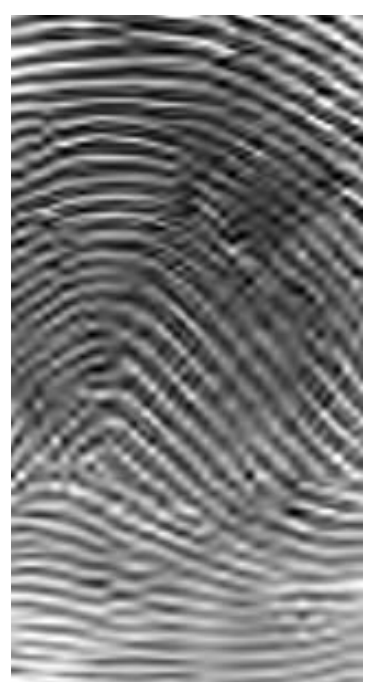

(b1)

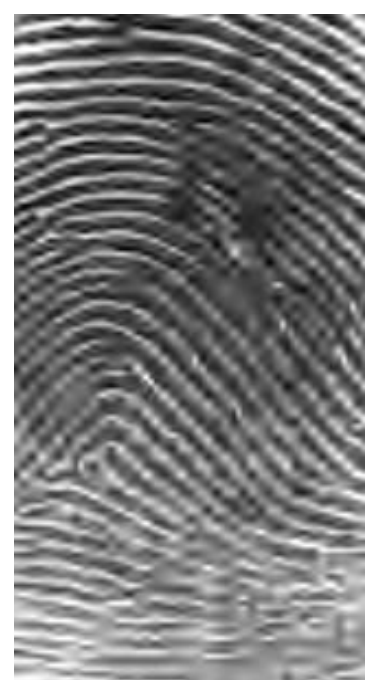

(c1)

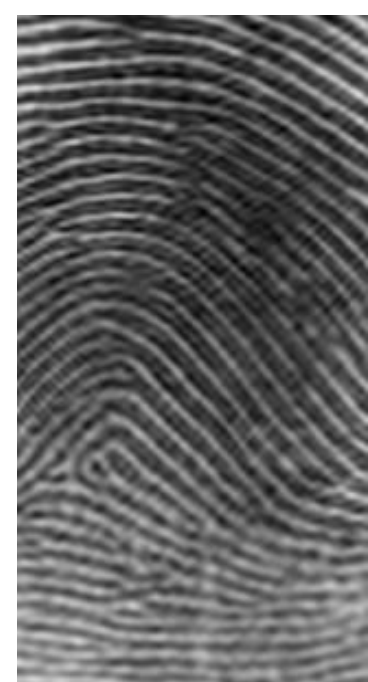

(d1)

Compressed images at compression ratio 103:1 with (a1) JPEG2000, (b1) WSQ, (c1) SPIHT and (d1) WAC

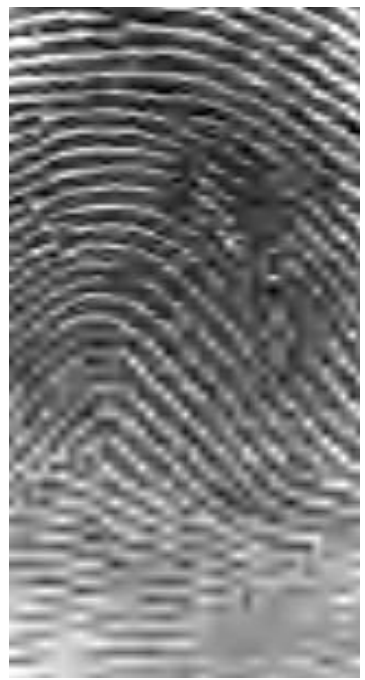

(a2)

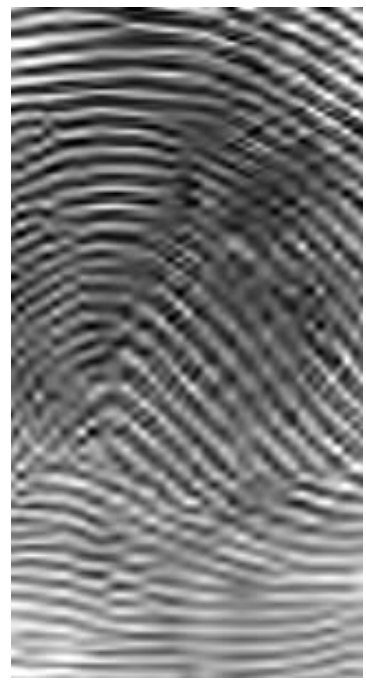

(b2)

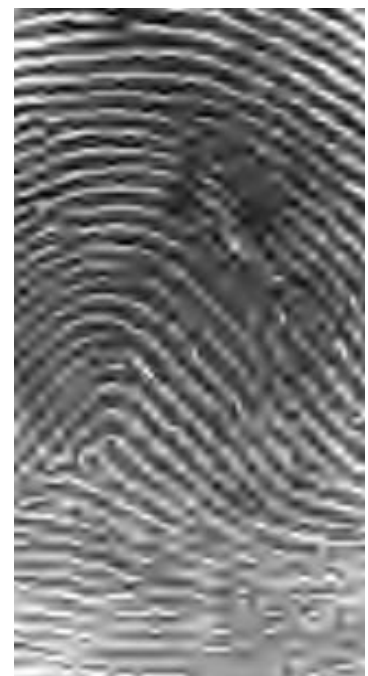

(c2)

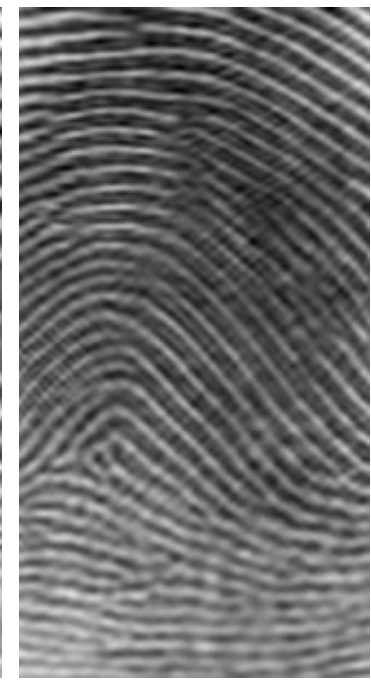

(d2)

Compressed images at compression ratio 133:1 with (a2) JPEG2000, (b2) WSQ, (c2) SPIHT and (d2) WAC

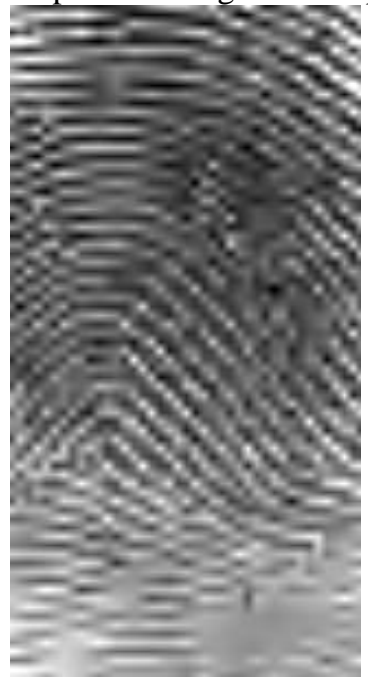

(a3)

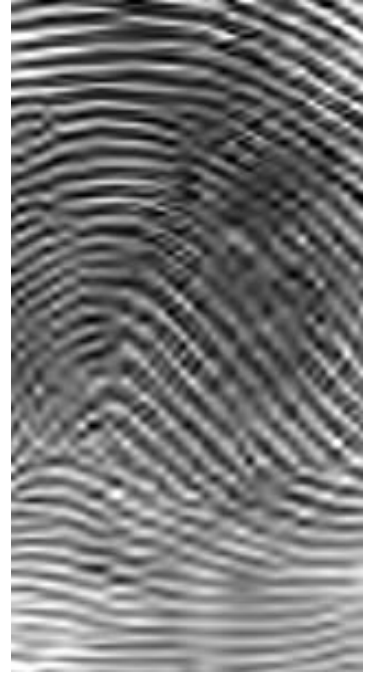

(b3)

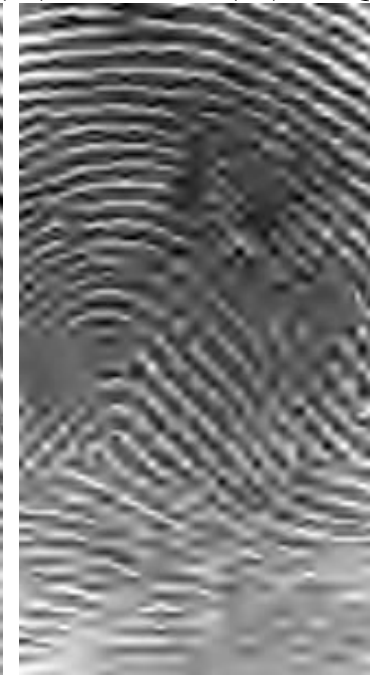

(c3)

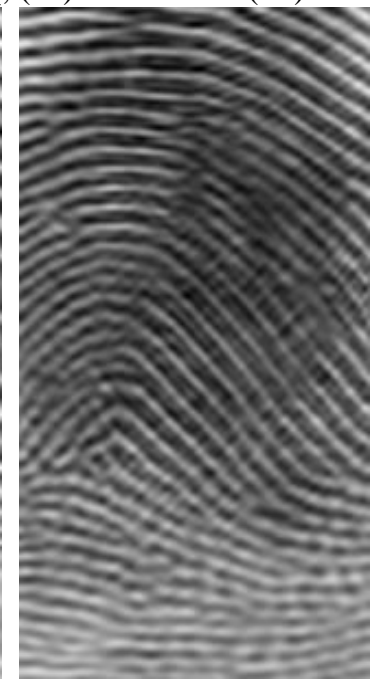

(d3)

Compressed images at compression ratio 182:1 with (a3) JPEG2000, (b3) WSQ, (c3) SPIHT and (d3) WAC

Figure 15-a. Arch compressed fingerprint images with (a) JPEG2000, (b) WSQ, (c) SPIHT and (d) the proposed WAC, at different compression ratio, (1) 103:1, (2) 133:1 and (3) 182:1. 


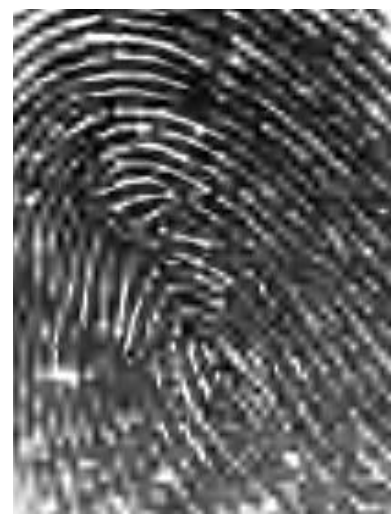

(a1)

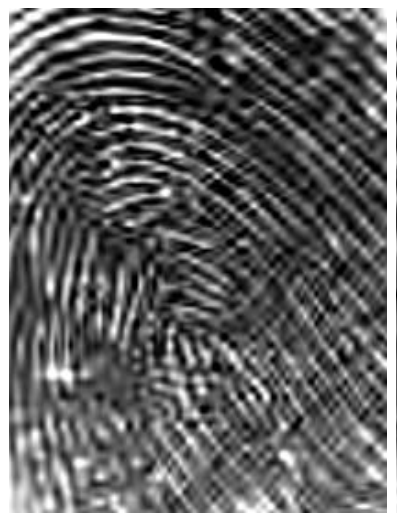

(b1)

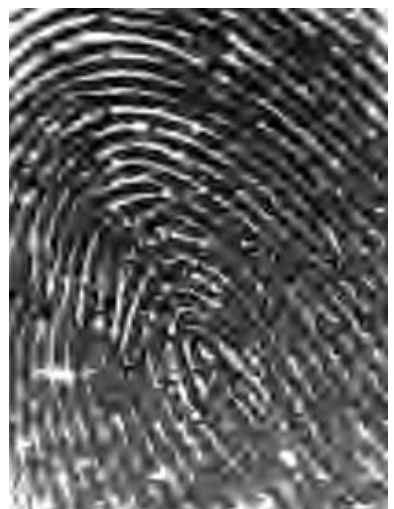

(c1)

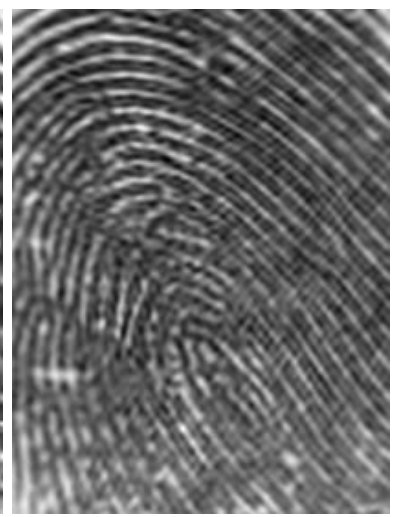

(d1)

Compressed images at compression ratio 103:1 with (a1) JPEG2000, (b1) WSQ, (c1) SPIHT and (d1) WAC

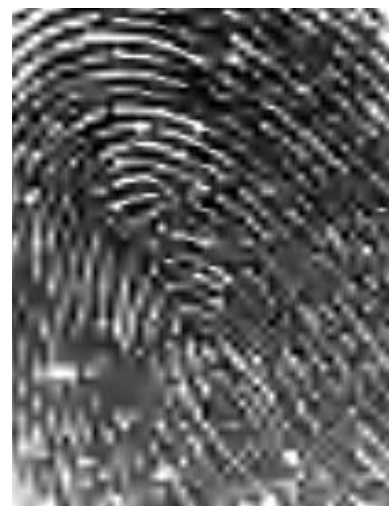

(a2)

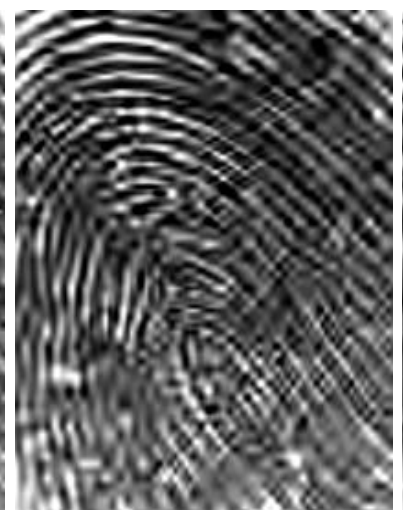

(b2)

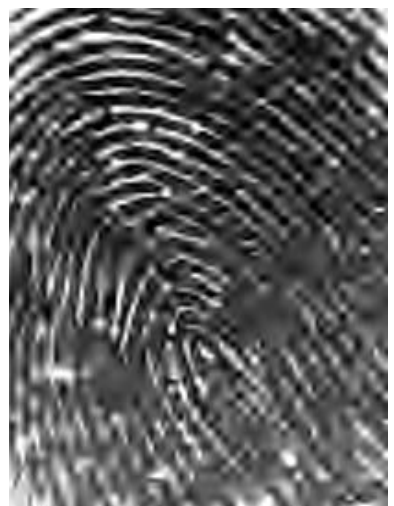

(c2)

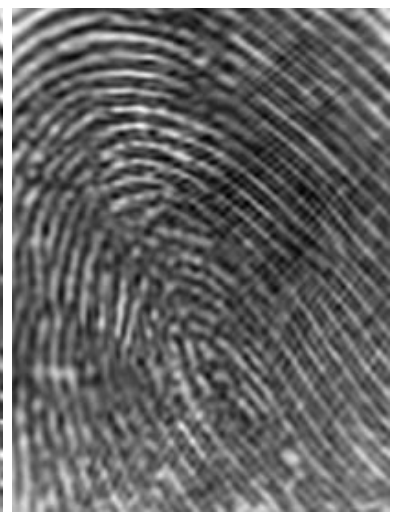

(d2)

Compressed images at compression ratio 133:1 with (a2) JPEG2000, (b2) WSQ, (c2) SPIHT and (d2) WAC

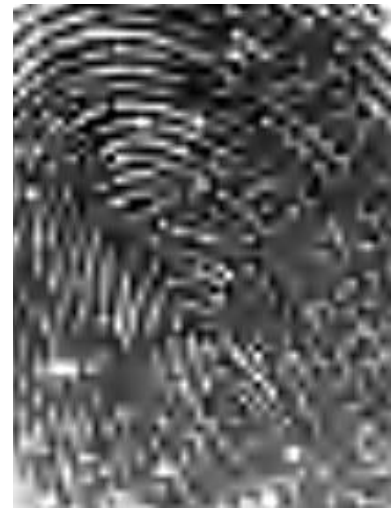

(a3)

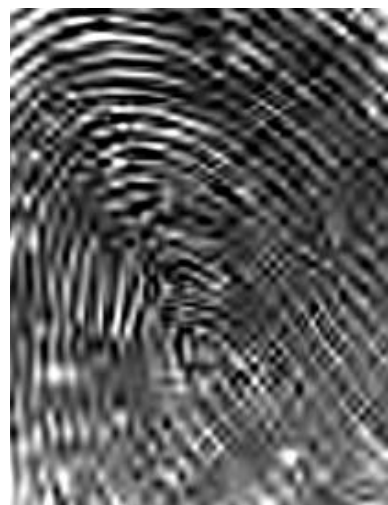

(b3)

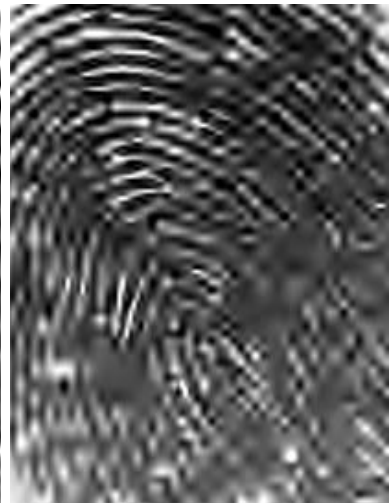

(c3)

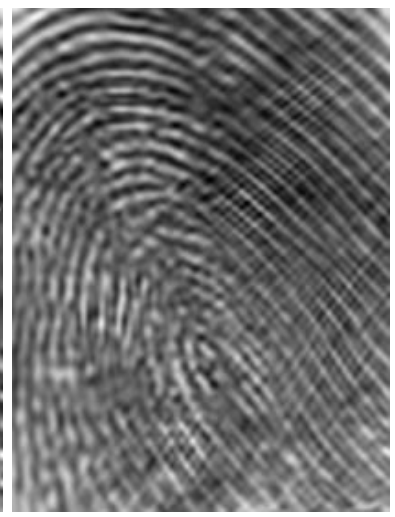

(d3)

Compressed images at compression ratio 182:1 with (a3) JPEG2000, (b3) WSQ, (c3) SPIHT and (d3) WAC

Figure 15-b. Right loop compressed fingerprint images with (a) JPEG2000, (b) WSQ, (c) SPIHT and (d) the proposed WAC, at different compression ratio, (1) 103:1, (2) 133:1 and (3) 182:1. 


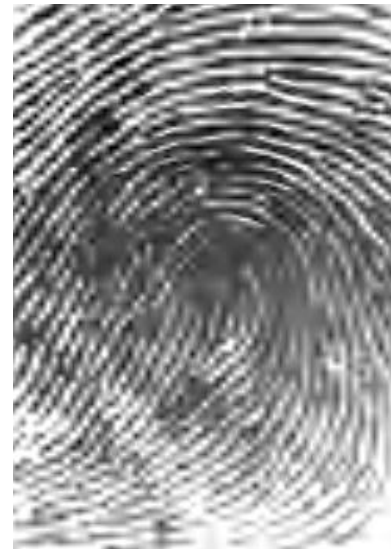

(a1)

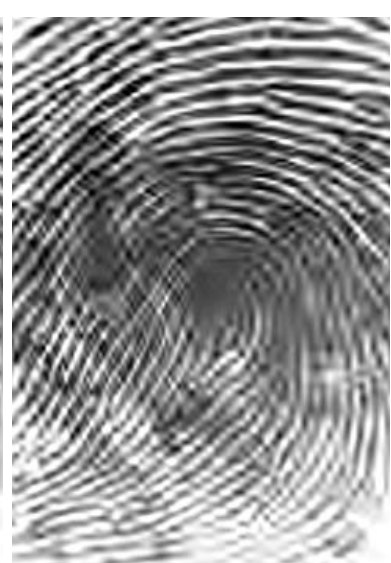

(b1)

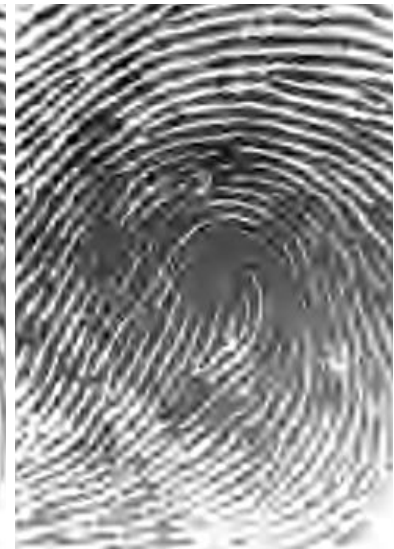

(c1)

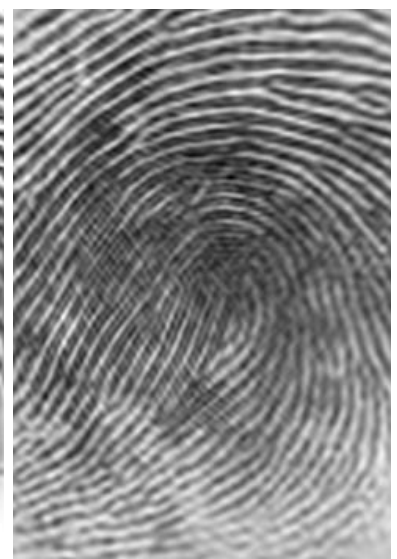

(d1)

Compressed images at compression ratio 103:1 with (a1) JPEG2000, (b1) WSQ, (c1) SPIHT and (d1) WAC

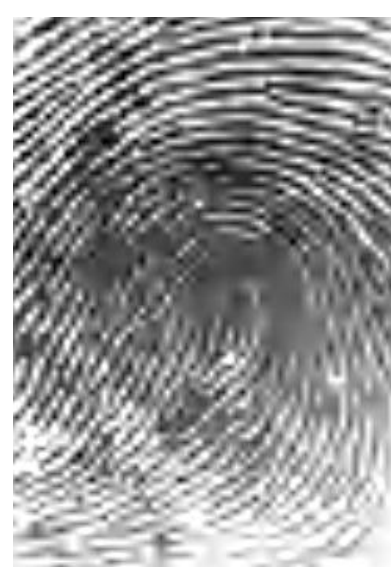

(a2)

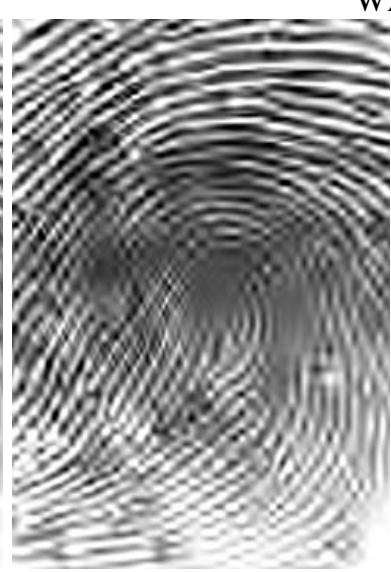

(b2)

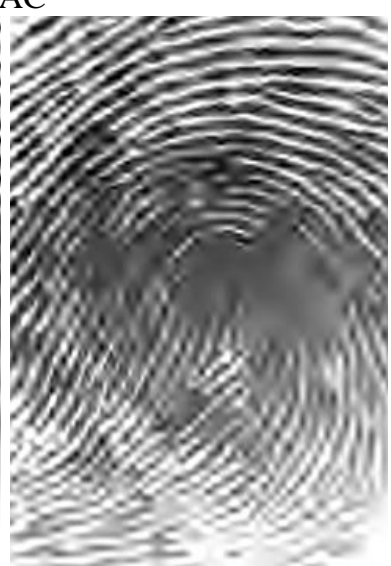

(c2)

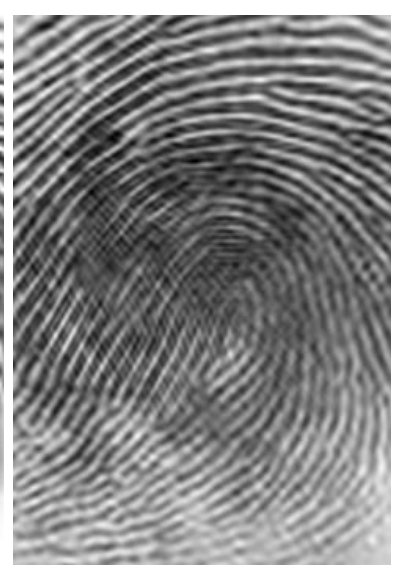

(d2)

Compressed images at compression ratio 133:1 with (a2) JPEG2000, (b2) WSQ, (c2) SPIHT and (d2)

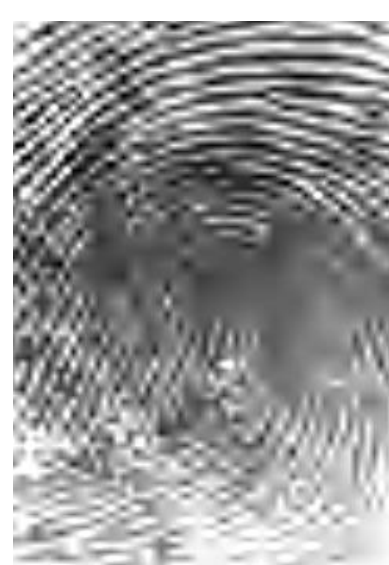

(a3)

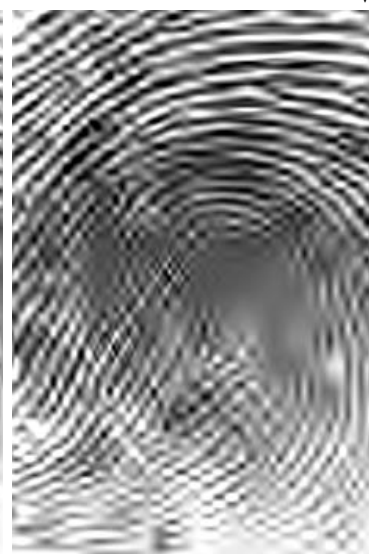

(b3)

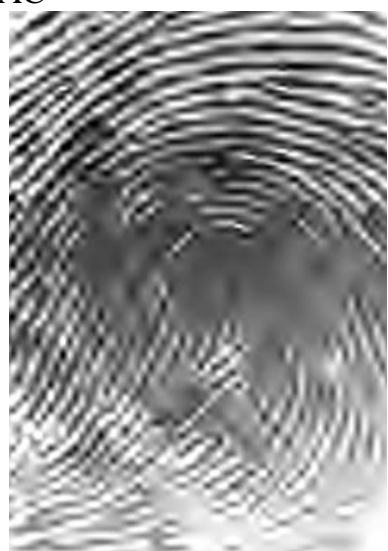

(c3)

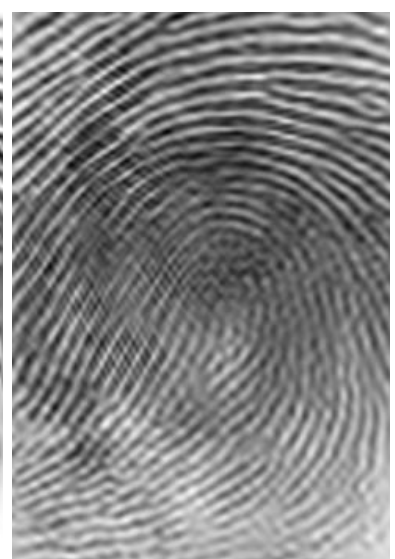

(d3)

Compressed images at compression ratio 182:1 with (a3) JPEG2000, (b3) WSQ, (c3) SPIHT and (d3) WAC

Figure 15-c. Left loop compressed fingerprint images with (a) JPEG2000, (b) WSQ, (c) SPIHT and (d) the proposed WAC, at different compression ratio, (1) 103:1, (2) 133:1 and (3) 182:1. 


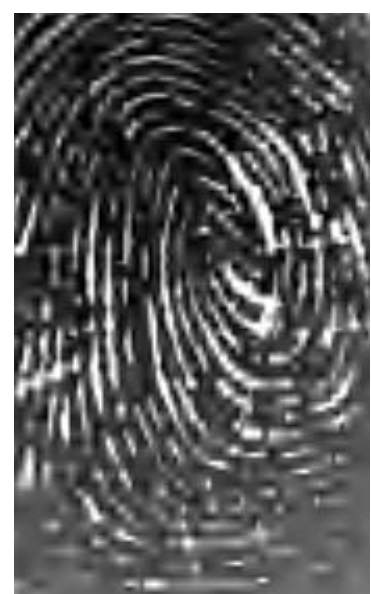

(a1)

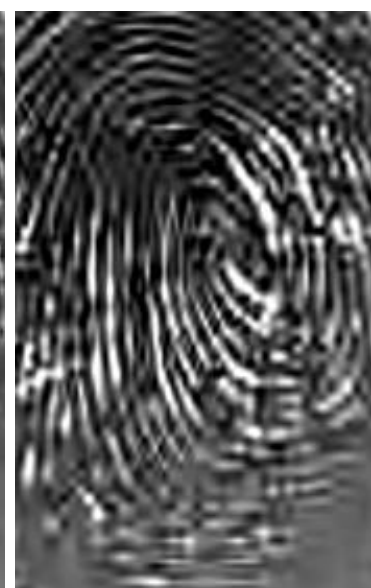

(b1)

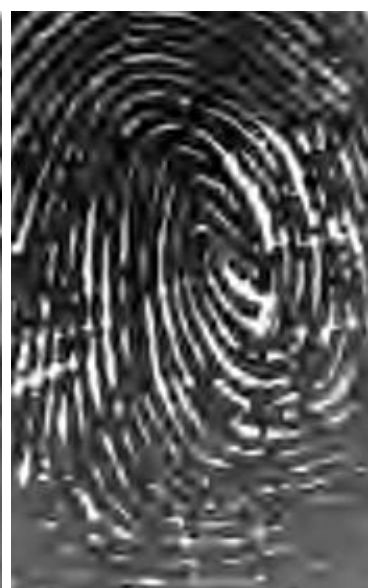

(c1)

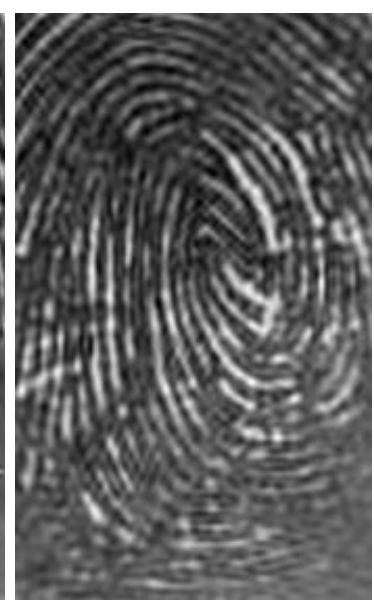

(d1)

Compressed images at compression ratio 103:1 with (a1) JPEG2000, (b1) WSQ, (c1) SPIHT and (d1)

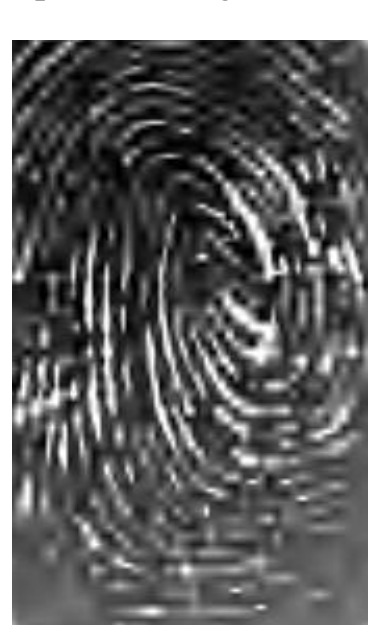

(a2)

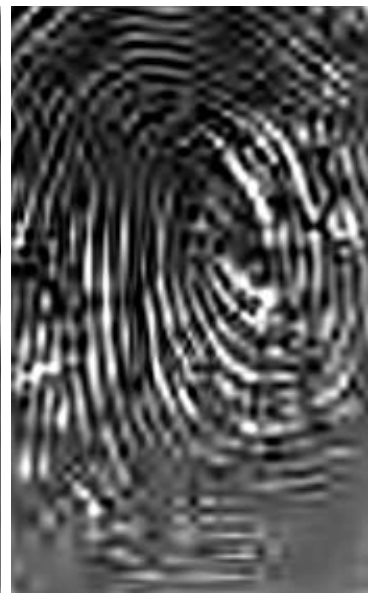

(b2)

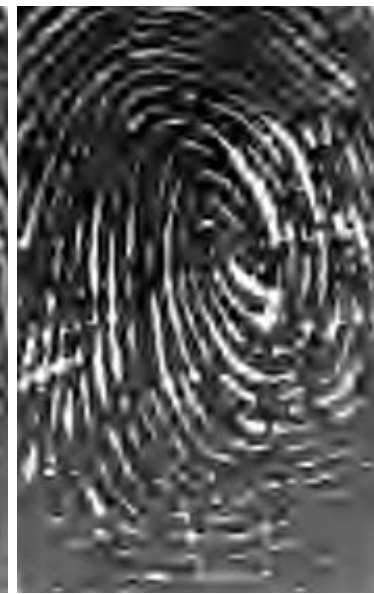

(c2)

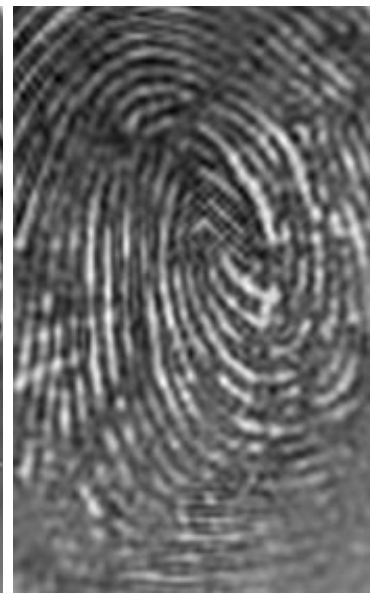

(d2)

Compressed images at compression ratio 133:1 with (a2) JPEG2000, (b2) WSQ, (c2) SPIHT and (d2) WAC

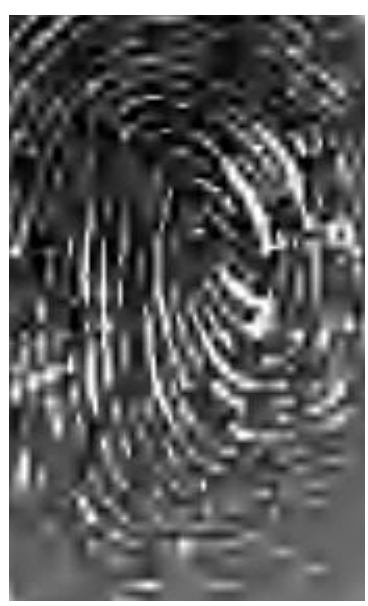

(a3)

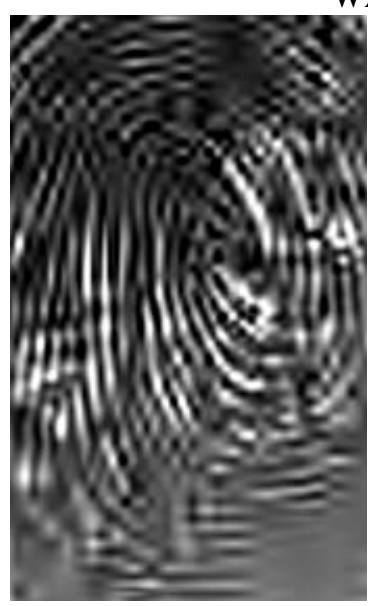

(b3)

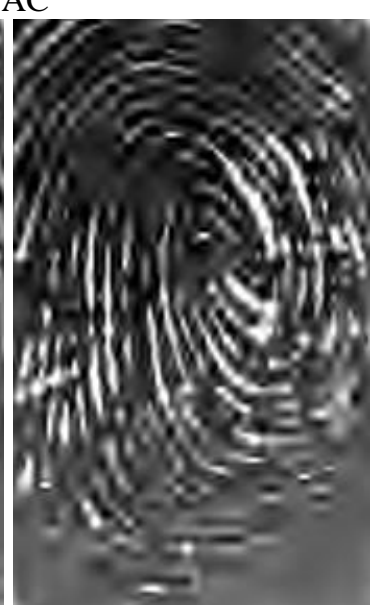

(c3)

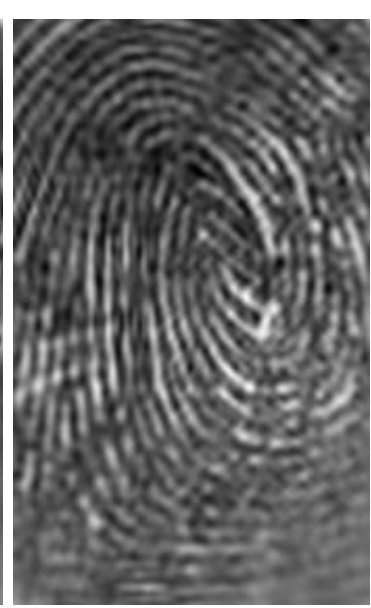

(d3)

Compressed images at compression ratio 182:1 with (a3) JPEG2000, (b3) WSQ, (c3) SPIHT and (d3) WAC

Figure 15-d. Volute compressed fingerprint images with (a) JPEG2000, (b) WSQ, (c) SPIHT and (d) the proposed WAC, at different compression ratio (1) 103:1, (2) 133:1 and (3) 182:1. 
We observe for WAC that the ridge curves are not altered until the compression ratio 103:1 is reached. At this compression ratio we observe a small visible smoothing effect along the ridges. This smoothing effect is more pronounced when the compression ratio increases. This smoothing effect does not represent a serious degradation compared to the blur effect and degradation observed in some areas with JPEG2000, SPIHT and WSQ compressions for the same compression ratio 103:1, 133:1 and 182:1.

From Figure 15, we observe that for all considered compression ratios; the proposed compression method gives better perceptual image quality compared to the others. These results confirm that the proposed image compression is appropriated to fingerprint images. It preserves the main structural features of fingerprint images.

\subsection{Image distortion control scheme}

The block diagram of Figure 16 shows the different stages of the proposed approach.

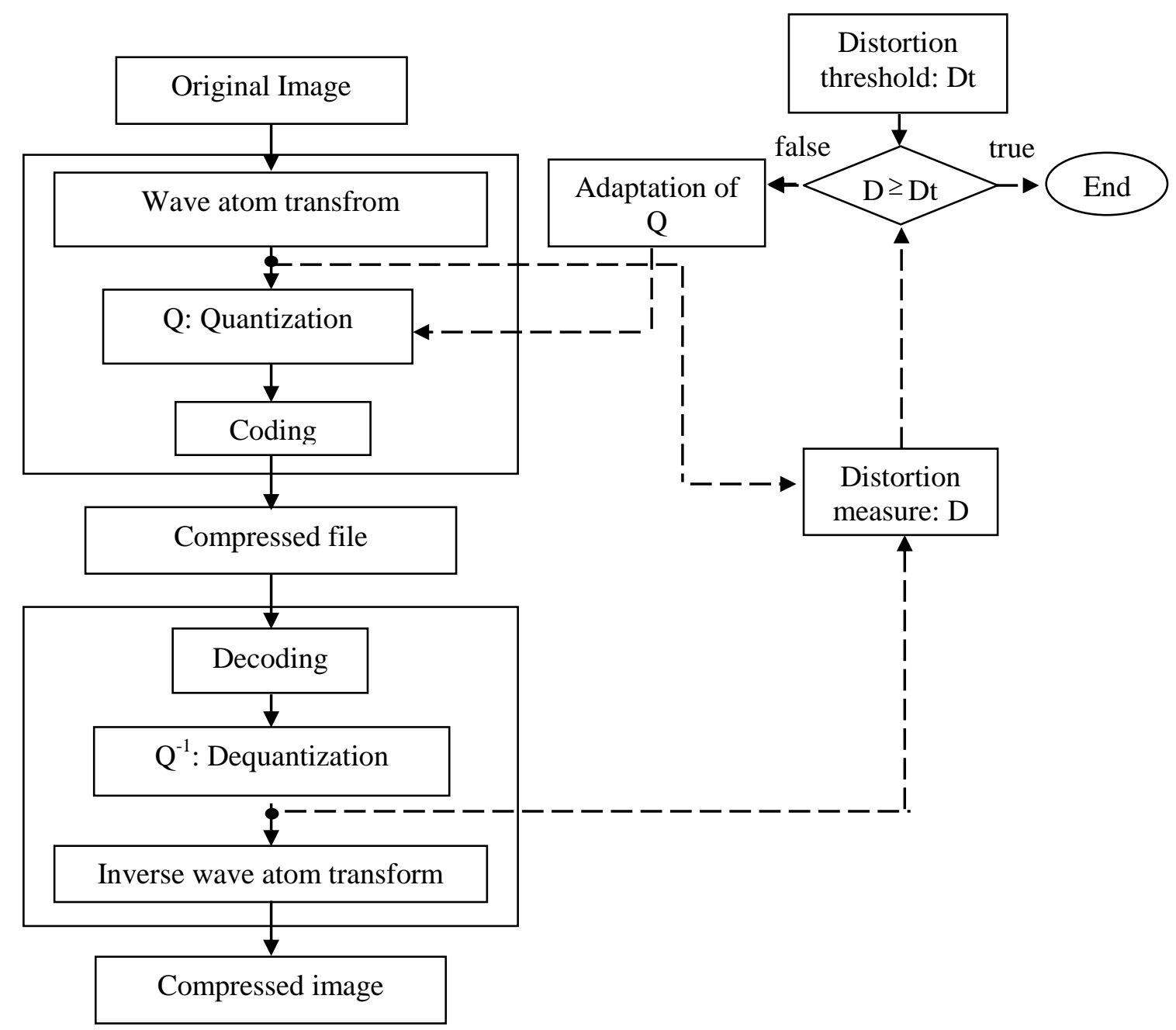

Figure 16. Block diagram of the proposed image distortion control process. 
The quantization step $\Delta_{i}$ corresponding to the subband $s_{i}$ is computed as follows:

$\alpha \Delta_{i}=\frac{\beta_{i}}{\log \left(\operatorname{var}\left(s_{i}\right)\right)}$

$\operatorname{var}\left(s_{i}\right)$ represents the coefficient variance in the subband $s_{i}$.

$\beta_{i}$ is a multiplicative coefficient corresponding to the subband $s_{i}$.

$\alpha$ is a multiplicative coefficient which is tuned according to the compression ratio and the image quality measure.

In the proposed compression approach, the number of quantization intervals depends on the desired compression ratio. This number could be adapted and controlled by the WAM. In the following we refer to it by $D$ resulting from coefficient selection and quantization process. Here, the image quality is used as an estimate of the distortion level. Thus, a low quality index corresponds to a good image quality whereas a high quality index corresponds to a low image quality level. The quantization step is then adjusted according to a predetermined distortion threshold $D_{t}$. For a given compression rate the number of quantization intervals is decreased, if $D<D t$, and increased in the other case.

It is worth noticing that the computation of the image quality threshold is a very crucial stage which depends strongly on the subsequent fingerprint image processing and the type of the used recognition approach. For example, we can choose a threshold that does not tolerate any visible distortion or another one that tolerates some degradation but without any consequence on the recognition system. Thus, the proposed method is very flexible and can adapt easily to the user need. It depends essentially on the recognition method to be used. Here, the threshold has been determined from an experimental validation and testing on several images. However, for future work, we propose to determine this threshold using a learning approach. From the experiments it is observed that for the same image quality measure WAM=0.14 the compression ratio can vary from 131:1 to $149: 1$ depending on the images of the database DB1 of FVC 2004 [45].

\subsection{Validation of the proposed compression scheme using a recognition system}

In this last part, we evaluate the performance of the proposed fingerprint compression system by means of fingerprint recognition system based on minutiae matching, which is still considered as the most widely used biometric approach for person identification.

As fingerprint database, we choose DB1 database of FVC2004 [45] which is an international fingerprint verification competition framework. This database is markedly more difficult than the other FVC databases, due to the distortions deliberately introduced. This database contains 80 fingerprint images. For the tests we apply the recognition system to check if it identifies individuals from their compressed fingerprint images.

As a fingerprint recognition system based on minutiae matching, we use a well-known fingerprint 
feature called adjacent orientation vector, or AOV [55]. This system has been used successfully in a highly secured and automated identification system for payroll tracking as well as authorized access to working areas. First, a possible minutiae pairs are found by AOV. After this, a preliminary matching is applied in order to ensure reliability followed by a fine matching to overcome possible distortion. This last step permits to make the algorithm more robust [55].

The procedure for the validation of the proposed compression method is to compress an image at different compression ratio and to determine when the compression is so strong that the recognition process fails in identifying the person. This ratio is finally compared to the rates given by other compression methods to see which method is the best compression.

The used database contains, for each individual, a set fingerprint images corresponding to various acquisitions of the same fingerprint. The test consists in selecting randomly a compressed fingerprint, decompress it and start the recognition system.

Figure 17 summarizes the recognition rate as a function of the compression ratio. The obtained results confirm that the recognition system using the images resulting from the proposed compression method WAC succeeds for all the compression ratios until 133:1, whereas those corresponding to the other compression standards yield low recognition performance for compression ratios higher than 103:1. Indeed, we observe that SPIHT based compression gives better results than JPEG2000 and this last gives better results than WSQ until a compression rate of 133:1. This result is consistent with the results of the image quality metric (Figure 14). This proves that the proposed image quality metric is consistent with the recognition system rate.

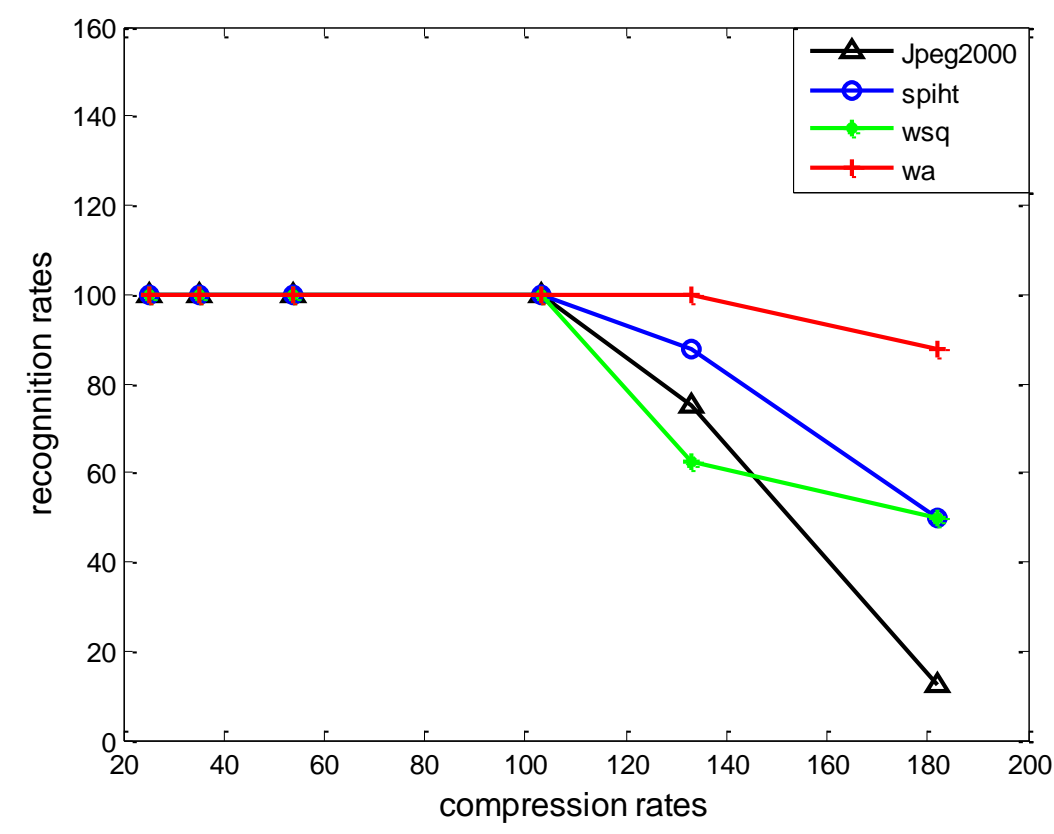

Figure 17. Recognition rates as a function of compression rates.

This validation by the recognition tests confirm the previous results and proves that the proposed 
compression method (WAC) based on Wave atom transform is better than the others compression standards: WSQ, JPEG2000, and SPIHT for fingerprint images.

\section{Conclusion}

The Wavelet transform is the basic tool in most image compression standards JPEG2000 and WSQ. This paper showed that for particular images, we can find a transform that is more appropriate than the classical Wavelets. In the first part of this work, we studied different transforms for several types of images, and we observed that Wavelet transform gives good results for several types of images except for fingerprint images for which Wave atom transform is better appropriate. Thereafter, we proposed a complete compression scheme based on this transform. A comparison study of compression efficiency revealed that the proposed method over performs the Curvelet based and the Wavelet based compression methods.

In order to control the compression performance, we proposed a perceptual metric based on Wave atom transform (WAM). The efficiency of the analysis and representation offered by Wave atom transform allows an efficient quantization of the fingerprint image. To develop an efficient image quality metric, some properties of the human visual system such as contrast masking and entropy masking which are incorporated in the design of the metric. The proposed image quality metric has been validated on LIVE and IVC databases where the results shown that WAM is consistent with the human judgment. Furthermore the distortion control procedure based on the human visual system where various masking effects are incorporated, allowed the control and adjustment of the quantization process.

The complete system has been tested in terms of recognition according to the FVC DB1 database usually used by the recognition systems. The obtained results confirm that the proposed method outperforms WSQ, JPEG2000 and SPIHT for fingerprint image compression.

As perspectives, investigations will focus on the encoding problem of the Wave atom coefficients in order to improve the performance of the developed compression method. Another issue to be explored is the extension of the proposed method to other biometric data such as iris and palm vein.

\section{References}

[1] W. K. Pratt, J. Kane, H. C. Andrews, "Hadamard transform image coding," Proceedings of the IEEE, vol. 57, no. 1, pp. 58-68, January 1969.

[2] R. J. Clarke, Transform Coding of images, Academic Press, London, 1985.

[3] W.K Pratt, W. H. Chen, "Slant transform image coding," IEEE transaction commun. Vol. COM-22, pp. 1075-1093, Aug. 1974.

[4] Rao, K. R. and P. Yip, Discrete Cosine Transform- Algorithms, Advantages and Applications, New York: Academic Press, 1990.

[5] W.-H. Chen, C. H. Smith, and S. Fralick, "A Fast Computational Algorithm for the Discrete Cosine Transform", IEEE Trans. on Communications 25, 1004-1009, Sep 1977. 
[6] D. S. Taubman, M. W. Marcellin, JPEG 2000: Image Compression Fundamentals, Standards, and Practice, New York: Kluwer International Series in Engineering and Computer Science, Nov. 2001.

[7] D. Salomon, Data compression, the complete reference, Fourth edition, Springer, 2007.

[8] J. Liang, C. Tu, T. D. Tran, "Optimal pre- and post-processing for JPEG2000 tiling artifact removal," Conference on information sciences and systems, The johns hopkins university, March 12-14, 2003.

[9] J. Liang, C. Tu, T. D. Tran, "Optimal block boundary pre/postfiltering for wavelet based image and video compression," IEEE transactions on image processing, Vol. 14, No. 12, pp. 21512158, December 2005.

[10] F. Friedrich, H. Führ, L. Demaret, "Beyond wavelets: New image representation paradigms," Survey article in document and image compression, M. Barni and F. Bartolini (eds), pp. 179-206, May 2006.

[11] "Special Issue on Image Quality Assessment," Signal Processing, Vol 70, 1998.

[12] Z. Wang, A. Bovik, H. Sheikh, E. Simoncelli, "Image quality assessment: from error visibility to structural similarity," IEEE Trans. Image Process. 13, 600-612, 2004.

[13] H. Sheikh, A. Bovik, L. Cormack, "No-reference quality assessment using natural scene statistics: JPEG2000," IEEE Trans. Image Process. 14, 1918-1927, 2005.

[14] Z. Wang, A.C. Bovik, B.L. Evans, "Blind measurement of blocking artifacts in images," ICIP, Vancouver, Canada, pp. 981-984, 2000.

[15] H. Sheikh, Z. Wang, L. Cormack, A. Bovik, "Blind quality assessment for JPEG2000 compressed images," ICIP, New York, pp. 1735-1739, 2002.

[16] Z. Wang, E. Simoncelli, "Reduced-reference image quality assessment using a waveletdomain natural image statistic model," Human Vision and Electronic Imaging X, Proceedings, vol. 5666, pp. 149-159, 2005.

[17] X. Gao, W. Lu and D. Tao, "Wavelet based contourlet in quallity evaluation of digital images," Neurocomputing, 72 (1-3) pp, 378-385, 2008.

[18] M. Liu, X. Yang, "Image quality assessment using contourlet transform," optical engineering, 48(10)107201, October 2009.

[19] S. Daly, "The visible differences predictor: An algorithm for the assessment of image fidelity," MIT Press, Cambridge, MA, 179-206.

[20] H.R. Sheikh, A.C. Bovik, G. de Veciana, "An information fidelity criterion for image quality assessment using natural scene statistics," IEEE Trans. Image Process. 14, 2117-2128, 2005.

[21] I. Avcibas, B. Sankur, K. Sayood, "Statistical evaluation of image quality measures," Journal of Electron Imaging 11, 206-213, 2002.

[22] E. Candes, D. Donoho, "Recovering edges iill-posed inverse problems: optimality of Curvelet," annals of statistics, vol. 30, no. 3, pp. 784-842, 2002.

[23] Z. Liu; L. J. Karam, A.B. Watson, "JPEG2000 encoding with perceptual distortion control," IEEE transactions on image processing; volume. 15; issue. 7, pp. 1763-1778, July 2006.

[24] Mallat, S. G., "A theory for multiresolution signal decomposition: The wavelet representation," IEEE Trans. On Pattern Analysis and Machine Intelligence, 674-693, July 1989.

[25] A. H. Tewfok, D. Sinha, and P. Jorgensen, "On the optimal choice of a wavelet for signal representation," IEEE Trans. Info. Theory, 38(2):747-765, 1992.

[26] J. Villasenor, B. Belzer, and J. Liao, "Wavelet filter evaluation for efficient image compression," IEEE Trans. On Image Processing, (4):1053-1060, 1995.

[27] Youssef, A., "Selection of good biorthogonal Wavelets for data compression," International Conference on Imaging, Science, Systems, and Technologiy, CISST '97, Las Vegas, pp. 323-330, June 1997.

[28] N. Ratha, R. Bolle, Automatic fingerprint recongnition systems, Springer, 2004.

[29] D. Maltoni, D. Maio, A. K. Jain, S. Prabhakar, Handbook of fingerprint recognition, corrected second printing, Springer, 2005.

[30] V. Chappelier, C. Guillemot, "Oriented Wavelet Transform for Image Compression and Denoising," IEEE Transactions on image processing, Volume: 15, Issue: 10 , Page(s): 2892 $2903,2006$. 
[31] H. T. Gabor, Fundamentals of Computerized Tomography: Image Reconstruction from Projections (2nd ed.), Springer, ISBN 978-1-85233-617-2, 2009.

[32] E. Candes, D. Donoho, "Ridgelets: A key to higher-dimensional intermittency?," Philosophical transactions Royal Society, Mathematical, physical and engineering sciences, vol. 357, no. 1760, pp.2495-2509, 1999.

[33] E. Candes, D. Donoho, "Curvelets - A surprisingly effective nonadaptive representation for objects with edges, curves and surfaces," Curves and Surfaces, Vanderbilt University Press, Nashville, TN, 1999.

[34] M. N. Do and M. Vetterli, "The contourlet transform: An efficient directional multiresolution image representation," IEEE Transactions on Image Processing, Oct. 2003. Volume: 14, Issue: 12, pp. 2091-2106.

[35] N. G. Kingsbury, "The dual-tree complex wavelet transform: a new efficient tool for image restoration and enhancement," in European Signal Processing Conference, pp. 319_322, Sept. 1998.

[36] A. B. Watson, "The cortex transform: rapid computation of simulated neural images," Computer Vision, Graphics, and Image Processing, vol. 39(3), pp. 311_327, Sept. 1987.

[37] E. P. Simoncelli and W. T. Freeman, "The steerable pyramid: A flexible architecture for multiscale derivative computation," in IEEE International Conference on Image Processing, Nov. 1995. , Vol. III, pp. 444-447.

[38] R.M. Merseraeau and A.V. Oppenheim, "Digital reconstruction of multidimensional signals from their projections," Proc. IEEE, 62, (1974), pp.1319-1338.

[39] L. Demanet, L. Ying, "Wave atom and sparsity of oscillatory patterns," Appl. Comput, vol. 23, Issue 3, pp. 368-387, 2007.

[40] S. Mallat, A Wavelet Tour of Signal Processing. Second edition. Academic Press, OrlandoSan Diego, 1999.

[41] J. P. Antoine, R. Murenzi, "Two-dimensional directional wavelets and the scale-angle representation," Sig. Process. 52, 259-281, 1996.

[42] I. Selesnick, R. G. Baraniuk, N. G. Kingsbury, "The dual-tree complex wavelet transform," IEEE Sig. Proc. Mag. 22(6) (2005) 123-151.

[43] L. Villemoes, Wavelet packets with uniform time-frequency localization, Comptes-Rendus Mathematique, 335-10 (2002) 793-796.

[44] Z. Haddad, A. Beghdadi, A. Serir, A. Mokraoui, " A new fingerprint image compression based on wave atoms transform," IEEE International symposium on signal processing and information technoloy, ISSPIT 2009, pp. 89-94, Ajman, December, 14-17, 2009.

[45] D. Maltoni, D. Maio, A.K. Jain, S. Prabhakar, Handbook of fingerprint recognition, Springer, London, 2009.

[46] A. B. Watson, R. Borthwick and M. Taylor, "Image quality and entropy masking," In Proc. SPIE, vol. 3016, 2-12, 1997.

[47] A. Ninassi, O. Le Meur, P. Le caller and D. Barba, "On the performance of human visual system based image quality assessment metric using wavelet domain," In proc. SPIE, vol. 6806, 680610-680610-12, 2008.

[48] http://live.ece.utexas.edu/

[49] Z. Wang and EP Simoncelli. "Translation insensitive image similarity in complex wavelet domain," ICASSP, Vol. 2, pp. 573-576, March 2005.

[50] X. Gao, W. Lu and D. Tao, "Wavelet based contourlet in quality evaluation of digital images," Neurocomputing, 72 (1-3) pp, 378-385, 2008.

[51] M. Poulos, A. Evangelou, E. Magkos, S. Papavlasopoulos, "Fingerprint verification based on image processing segmentation using an onion algorithm of computational geometry," in WSPC Proceedings, February 21, 2004.

[52] Woo Kyu Lee; Jae Ho Chung, "Automatic real-time identification of fingerprint images using wavelet transform and gradient of Gaussian," in IEEE Asia Pacific Conference on Circuits and Systems, pp. $508-511,1996$.

[53] Chih-Jen Lee; Sheng-De Wang, "Fingerprint feature extraction using Gabor filters," in Electronics Letters, Vol. 354 , pp. 288 -290, 18 Feb. 1999.

[54] M. D. Adams, JasPer Software Reference Manual, ISO/IEC JTC 1/SC 29/WG 1N 2415, 2006. 
[55] G. S. Ng, X. Tong, X. Tang and D. Shi, "Adjacent orientation vector based fingerprint minutiae matching system", Pattern Recognition, ICPR 2004, Proceedings of the 17th International Conference on, volume. 1; pp. 528-531, 2004.

[56] E. J. Candès, L. Demanet, D. L. Donoho and L. Ying. Fast discrete curvelet transforms. Multiscale Model. Simul., 5 861-899 (2006).

[57] Patrick Le Callet, Florent Autrusseau, "Subjective quality assessment IRCCyN/IVC database", 2005.

[58] D. M. Chandler and S. S. Hemami, "VSNR: A Wavelet-based visual signal to noise ratio for natural images, " IEEE Trans. Image Process. 16(9) 2284-2298, 2007.

[59] http://foulard.ece.cornell.edu/dmc27/vsnr/vsnr.html

[60] T. Chen, X. Jiang, W-Y. Yau, " Fingerprint image quality analysis", IEEE International Conference on Image Processing, ICIP 2004: 1253-1256

[61] H. Fronthaler, K. Kollreider, J. Bigun, J. Fierrez, F. Alonso-Fernandez, J. Ortega-Garcia, J.Gonzalez-Rodriguez. "Fingerprint image-quality estimation and its application to multialgorithm verification. IEEE Trans. IFS, 3(2): 331-338, 2008. 